\title{
ArcheoSciences
}

Revue d'archéométrie

41-1 | 2017

Varia

\section{Analyse technologique, étude de provenance et datation par le radiocarbone du dépôt de demi- produits ferreux de Durrenentzen (Haut-Rhin, France) : une vision renouvelée de l'économie du fer au premier âge du Fer}

Technological analysis, provenance study and radiocarbon dating of iron bipyramidal semi-products of the Durrenentzen deposit (Haut-Rhin, France): A renewed vision of the iron economy during Iron Age I

Marion Berranger, Sylvain Bauvais, Mostepha Boukezzoula, Stéphanie Leroy, Alexandre Disser, Enrique Vega, Michel Aubert, Philippe Dillmann et Philippe Fluzin

\section{(2) OpenEdition}

Journals

Édition électronique

URL : https://journals.openedition.org/archeosciences/4883

DOI : 10.4000/archeosciences.4883

ISSN : 2104-3728

Éditeur

Presses universitaires de Rennes

Édition imprimée

Date de publication : 21 juin 2017

Pagination : 45-67

ISBN : 978-2-7535-5890-8

ISSN : 1960-1360

\section{Référence électronique}

Marion Berranger, Sylvain Bauvais, Mostepha Boukezzoula, Stéphanie Leroy, Alexandre Disser, Enrique Vega, Michel Aubert, Philippe Dillmann et Philippe Fluzin, « Analyse technologique, étude de provenance et datation par le radiocarbone du dépôt de demi-produits ferreux de Durrenentzen (HautRhin, France) : une vision renouvelée de l'économie du fer au premier âge du Fer », ArcheoSciences [En ligne], 41-1 | 2017, mis en ligne le 21 juin 2019, consulté le 28 janvier 2022. URL : http:// journals.openedition.org/archeosciences/4883; DOI : https://doi.org/10.4000/archeosciences.4883 


\title{
Analyse technologique, étude de provenance et datation par le radiocarbone du dépôt de demi-produits ferreux de Durrenentzen (Haut-Rhin, France) : une vision renouvelée de l'économie du fer au premier âge du Fer
}

\author{
Technological Analysis, Provenance Study and Radiocarbon Dating of Iron \\ Bipyramidal Semi-products of the Durrenentzen Deposit (Haut-Rhin, France): \\ A Renewed Vision of the Iron Economy during Iron Age I
}

\author{
Marion Berranger ${ }^{\mathrm{a}}$, Sylvain Bauvais ${ }^{\mathrm{a}, \mathrm{b}}$, Mostepha Boukezzoula ${ }^{\mathrm{a}}$, \\ Stéphanie Leroy ${ }^{\mathrm{a}, \mathrm{b}}$, Alexandre Disser ${ }^{\mathrm{a}, \mathrm{b}}$, Enrique VeGA ${ }^{\mathrm{a}, \mathrm{b}}$, Michel AuberT ${ }^{\mathrm{a}}$, \\ Philippe Dillmann ${ }^{\mathrm{a}}$ b et Philippe Fluzin ${ }^{\mathrm{a}}$
}

\begin{abstract}
Résumé : Durant les premiers temps de la métallurgie du fer en Europe occidentale, le type de demi-produit le plus répandu est de forme bipyramidée. Bien que plus d'un millier de pièces soit connu, leurs conditions de fabrication et de circulation et même leur datation restent mal appréhendées Une approche interdisciplinaire a ainsi été appliquée au dépôt de demi-produits bipyramidés de Durrenentzen (Haut-Rhin), le troisième en Europe par son importance numérique, afin de reconstituer le contexte technologique, social et économique qui a conduit à son abandon. L'étude morphométrique des cinquante et un bipyramidés révèle une grande homogénéité globale du lot malgré des variations de détail. Quatre objets ont été sélectionnés pour études archéométriques. Les analyses métallographiques mettent en évidence des différences internes significatives en ce qui concerne la qualité des matériaux, la nature de l'alliage et les techniques de fabrication. Les analyses chimiques révèlent également des signatures différenciées. Enfin, des analyses radiocarbones réalisées sur le carbone contenu dans les aciers permettent de rattacher ce lot au premier âge du Fer. Les résultats de l'étude de ce dépôt exceptionnel permettent ainsi d'implanter le premier jalon d'une analyse plus générale modifiant significativement la perception de l'économie du fer au premier âge de Fer.
\end{abstract}

\begin{abstract}
During the early stages of the iron metallurgy in Western Europe, the most wide-spread type of semi-product is of bipyramidal shape. Even if their occurrence is frequent their conditions of manufacturing and of circulation and even their dating remain poorly known. Thus an interdisciplinary approach was applied to the Durrenentzen deposit (Haut-Rhin) - the third in Europe by its numerical importance - in order to reconstitute the technological, social and economic context which led to its abandonment. The morphometrical study of fifty one bipyramidal semi-products reveals a large overall homogeneity of the set in spite of detail variations. Four objects were selected for archaeometrical studies. The metallographic analyses highlight
\end{abstract}

\footnotetext{
"Laboratoire "Métallurgies et Cultures" - IRAMAT, UMR5060, CNRS, Université de Technologie de Belfort-Montbéliard, 90010 BELFORT Cedex. (marion.berranger@utbm.fr) (boukezzoula.mostepha@free.fr)(michel.aubert@utbm.fr)(philippe.fuzin@utbm.fr)

${ }^{b}$ Laboratoire Archéomatériaux et Prévision de l'Altération - IRAMAT, NIMBE, UMR3685, CEA, CNRS, Université Paris-Saclay, 91 191 GIF-SURYVETTECedex. (sylvain.bauvais@cea.fr) (stephanie.leroy@cea.fr) (alexandre.disser@cea.fr) (enrique.vega@cea.fr) (philippe.dillmann@cea.fr)
} 
significant internal differences regarding the quality of the materials, the nature of the alloy and the manufacturing techniques. The chemical analyses also reveal differentiated chemical signatures. Radiocarbon analyses realized on the carbon contained in steel allow finally to connect this deposit to the first Iron Age. Results about this exceptional deposit allow to implant the first milestone of a more general analysis modifying significantly the perception of the iron economy during the first Iron Age.

Mots clés : Analyse technologique, Étude de provenance, Datation par le radiocarbone, Demi-produit en fer, Âge du Fer, Alsace.

Keywords: Technological analysis, Provenance study, Radiocarbon dating, Iron semi-product, Iron Age, Alsace.

\section{INTRODUCTION}

En Europe occidentale, durant les premiers temps de la métallurgie du fer, l'un des principaux types de demi-produits connus est de forme bipyramidée. Un récent travail (Berranger, 2014) en recense ainsi plus de 1300 exemplaires issus de plus de 200 sites répartis entre la Grande-Bretagne et la Pologne. Leur zone principale de concentration couvre essentiellement le Nord-Est de la France (pour la France plus de 450 objets provenant d'une soixantaine de sites sont inventoriés) et l'ouest de l'Allemagne (environ 650 objets, dits "Spitzbarren » dans la littérature allemande, proviennent de 130 sites). Bien que leur occurrence soit fréquente, les conditions de fabrication, les modalités de circulation et même la datation de ces objets restent mal appréhendées. En effet, ils sont principalement découverts hors de leur contexte fonctionnel, dans la quasi-intégralité des cas en dépôts dits isolés, c'est-à-dire à l'écart de toute occupation humaine avérée et sans autre mobilier associé. Que les motivations de leur abandon aient été économiques (hypothèse de la cachette de forgeron) ou rituelles (dépôts dits votifs), elles ont conduit à l'abandon de quantités de métal considérables. La masse d'environ 450 bipyramidés a été relevée. Ils totalisent à eux seuls 2,1 tonnes de fer transformé. En estimant une masse déduite des objets actuellement connus, ce serait ainsi près de 6 tonnes de fer qui auraient été abandonnées sous la forme de ces demiproduits bipyramidés. Ils sont donc des témoins incontournables pour mieux saisir l'organisation et la circulation des productions métalliques durant des périodes précoces. En effet, bien que mal datés par le contexte archéologique, ils ont été attribués au premier âge du Fer (Berranger, 2014), au second âge du Fer (Senn et al., 2014, p. 159) et à l'époque gallo-romaine (Kleeman, 1981).

Les récents apports des approches archéométriques sur les études de procédés (Bauvais et Fluzin, 2009; Dillmann et al., 2015; Fluzin et al., 2011; Fluzin et al., 2012), les études de provenance (Desaulty et al., 2009; Leroy et al., 2012a; Leroy et al., 2014; Disser et al., 2014; Disser et al., 2016) et sur la datation des métaux ferreux (Leroy et al., 2012b; Leroy et al., 2015a; Leroy et al., 2015b) permettent aujourd'hui d'envisager d'éclairer plus avant les questions en suspens. Ces analyses permettent ainsi de déterminer les différents niveaux de spécialisation des artisans, en caractérisant par exemple les savoir-faire investis dans la fabrication de ces produits intermédiaires. Comme nous le verrons par la suite, leur étude chimique inclusionnaire rend également possible la détermination d'une segmentation ou d'une continuité entre les différentes étapes de la chaîne opératoire (notamment entre étapes de réduction et de fabrication de demiproduits). À terme, le recours à ces approches vise également à déterminer si ces objets proviennent d'un ou de plusieurs centres de production, afin de mieux saisir leurs conditions de fabrication et d'échanges.

Ces méthodologies interdisciplinaires ont été appliquées à l'étude de l'un des plus importants dépôts de bipyramidés connus (le troisième recensé en Europe continentale pour son importance numérique), celui de Durrenentzen (HautRhin, France), situé dans la zone de plus forte concentration de ce type de demi-produit (figure 1). La possibilité de caractériser une série de demi-produits de même type et d'un même contexte a motivé la reprise d'une caractérisation interdisciplinaire de cet ensemble exceptionnel. Les objectifs étaient multiples :

- déterminer la nature des matériaux et les techniques de fabrication des demi-produits;

- mettre en évidence, d'un point de vue structural, les spécificités et points communs entre ces objets issus d'un même lot;

- caractériser la composition chimique des inclusions de scories piégées dans le métal afin d'étudier leur origine;

- dater les objets eux-mêmes, par la méthode radiocarbone, étant donné qu'ils ne peuvent être datés par le seul contexte (absence de mobilier associé). 
Figure 1 : Carte de répartition des demi-produits bipyramidés en fer, par nombre d'objets découverts, et localisation du dépôt de Durrenenzten (pastille gris foncé). Figure 1: Distribution map of iron bipyramidal semi-products, by number of objects discovered, and localization of the Durrenenzten deposit (dark grey dot).

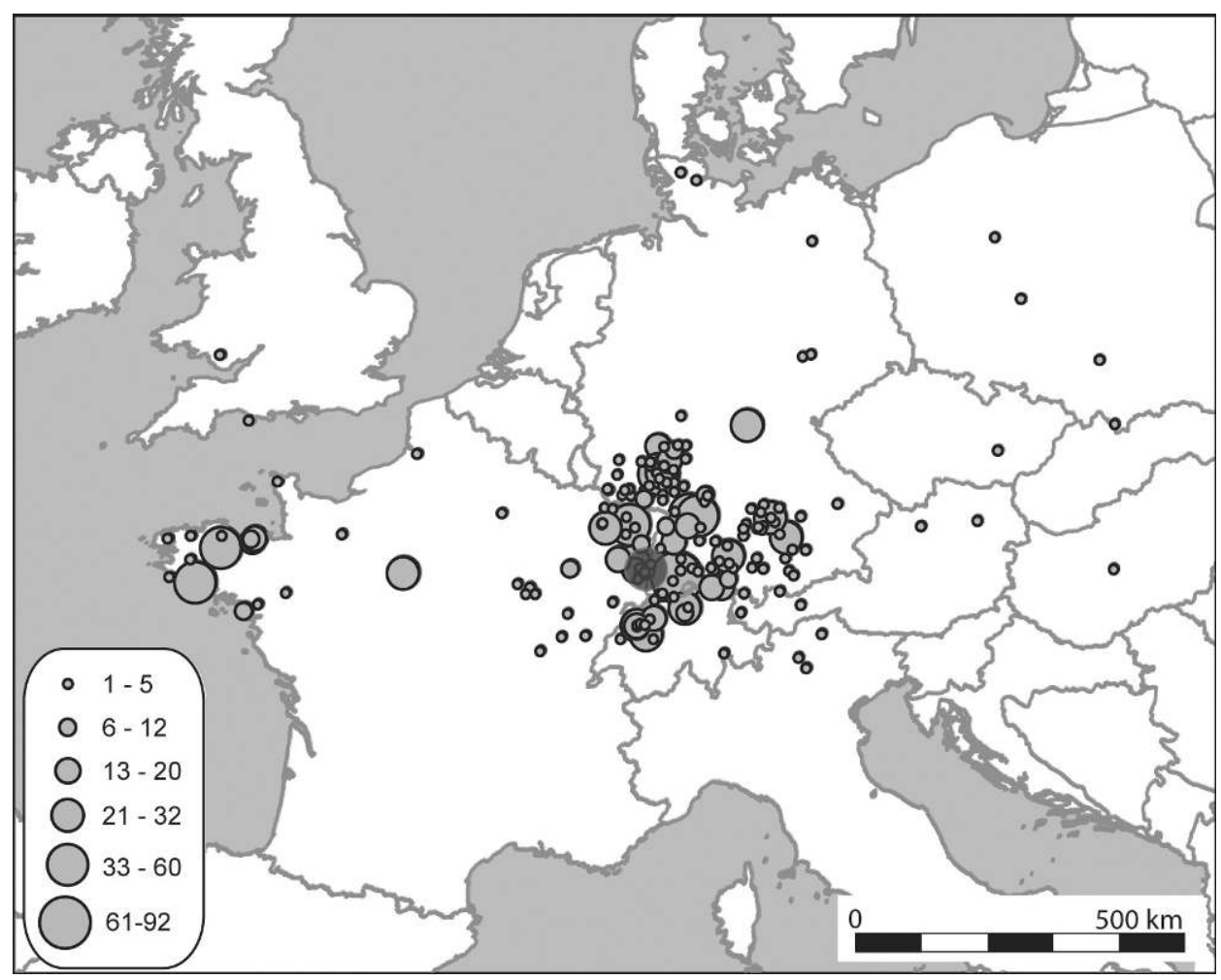

\section{Présentation géNérale des déCouvertes}

Cinquante et un demi-produits bipyramidés isolés de tout indice de datation ont été fortuitement découverts sur la commune de Durrenentzen en 1983, à l'occasion du creusement d'une tranchée de canalisation.

En 2015, un nouveau dépôt de quatorze lingots bipyramidés a été mis au jour à l'occasion d'un diagnostic de l'INRAP (Kuhnle, 2015) à une centaine de mètres du premier dépôt. La zone était vierge de tout autre indice archéologique sur les 1,8 ha sondés (les résultats de la fouille menée par la société Evéha durant l'été 2016 sont en cours d'exploitation).

Une première publication datant de 1986 (Biellmann, 1986) présente le contexte de découverte, les caractéristiques macroscopiques du lot ainsi que les résultats d'analyses métallurgiques menées sur deux objets (DUR.3 et DUR.6, analysés par l'APAVE alsacienne). Néanmoins, les conclusions indiquent que les objets ont été coulés dans un moule, et que les impuretés telles que le soufre ou le phosphore ont été extraits par affinage. Au vu des connaissances actuelles de la recherche sur les métallurgies anciennes, ces déductions ne peuvent que s'avérer erronées.

Les cinquante et un demi-produits sont d'un type massif, aux extrémités resserrées formées par un amincissement progressif de l'épaisseur, sans qu'elles aient été étirées. Les sections sont carrées ou rectangulaires. Ils se rattachent à la variante BCS1 (type court à section carrée et extrémités resserrées - Berranger, 2014) des bipyramidés retrouvés en France. Il s'agit d'une des variantes les plus fréquentes.

Les demi-produits ne laissent pas discerner, en surface, d'importantes porosités ou fissures permettant de supposer un mauvais compactage, au moins superficiel. Certains objets comportent des cavités rectilignes peu profondes (env. $10 \mathrm{~mm}$ de large sur 1 à $2 \mathrm{~mm}$ de profondeur) dont la présence est difficilement compréhensible. Biellmann les interprète comme des traces des cordelettes ayant maintenu l'ensemble, mais il est peu vraisemblable qu'elles aient marqué le métal en profondeur. Plusieurs objets ont une extrémité manquante, apparemment volontairement fragmentée dans l'optique de se conformer à un standard de masse ou de longueur.

La masse des demi-produits varie de 5500 à $6915 \mathrm{~g}$, avec une moyenne à $6049 \mathrm{~g}$, et la longueur est comprise entre 215 et $331 \mathrm{~mm}$ (figure 2 - métrologie détaillée dans Biellmann, 1986). L'exemplaire le plus petit est l'un de ceux comprenant une extrémité fragmentée. On constate une forte homogénéité globale des demi-produits, à la fois en termes de masses, longueurs, largeurs et épaisseurs, avec de modestes variations morphologiques de détail. Parmi ces détails, certains critères morphologiques attestent de fini- 
tions différentes, en lien possible avec des individus ou des ateliers distincts. Une première tentative de typologie est ici proposée en prenant en compte la forme des arêtes centrales
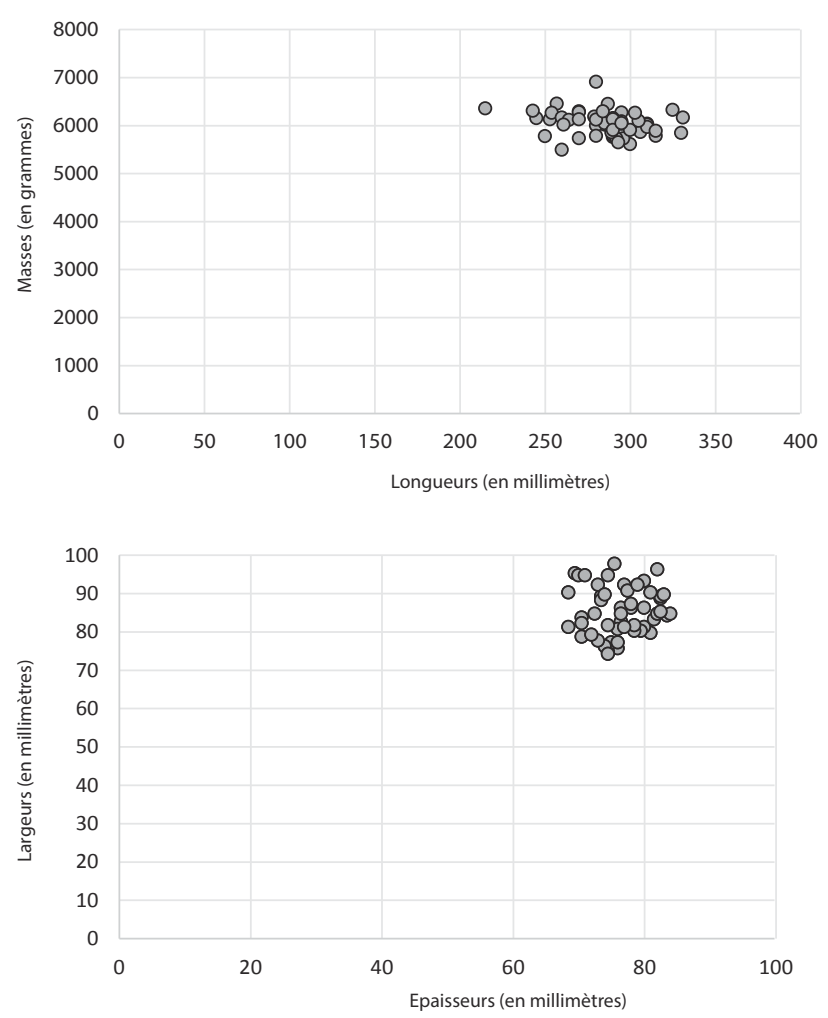

Figure 2 : Caractéristiques métrologiques des demi-produits en fer de Durrenentzen.

Figure 2: Metrological characteristics of the semi-products from the iron Durrenentzen deposit. et latérales (figure 3). Quatre grands types semblent pouvoir être distingués, même s'il est parfois délicat d'attribuer un type unique à chacun des demi-produits composant le dépôt. Toujours est-il que parmi les types morphologiquement les plus éloignés, les différences sont flagrantes. Il s’agit des demi-produits de type 1 dont la forme générale revêt le mieux le qualificatif de bipyramidé et de ceux de type 4 dont la morphologie est plus proche de ce que l'on qualifie de "saumon " dans d'autres régions du monde (Pleiner et Bjorkman, 1974).

Sur la base de cette première typologie et en raison des contraintes imposées par la conservation du mobilier archéologique, seuls quatre exemplaires ont été sélectionnés pour analyses. Deux avaient déjà été tronçonnés pour les besoins des analyses des années 1980 (DUR.3 et DUR.6). Malgré tout, cette étude représente un unicum en termes de nombre de demi-produit analysé de ce type (métallographie et chimie).

\section{MÉTHOdologie ET MOYENS EXPÉRIMENTAUX}

\section{Approches métallographiques}

Les quatre demi-produits : DUR.3, DUR.6, DUR.40 et DUR.48 ont subi dans un premier temps une analyse métallographique. L'objectif est d'établir la qualité des matériaux, d'évaluer de manière qualitative la composition chimique (fer/acier/phosphore) ainsi que de reconnaitre les techniques de fabrication employées (mise en évidence de soudures etc.).

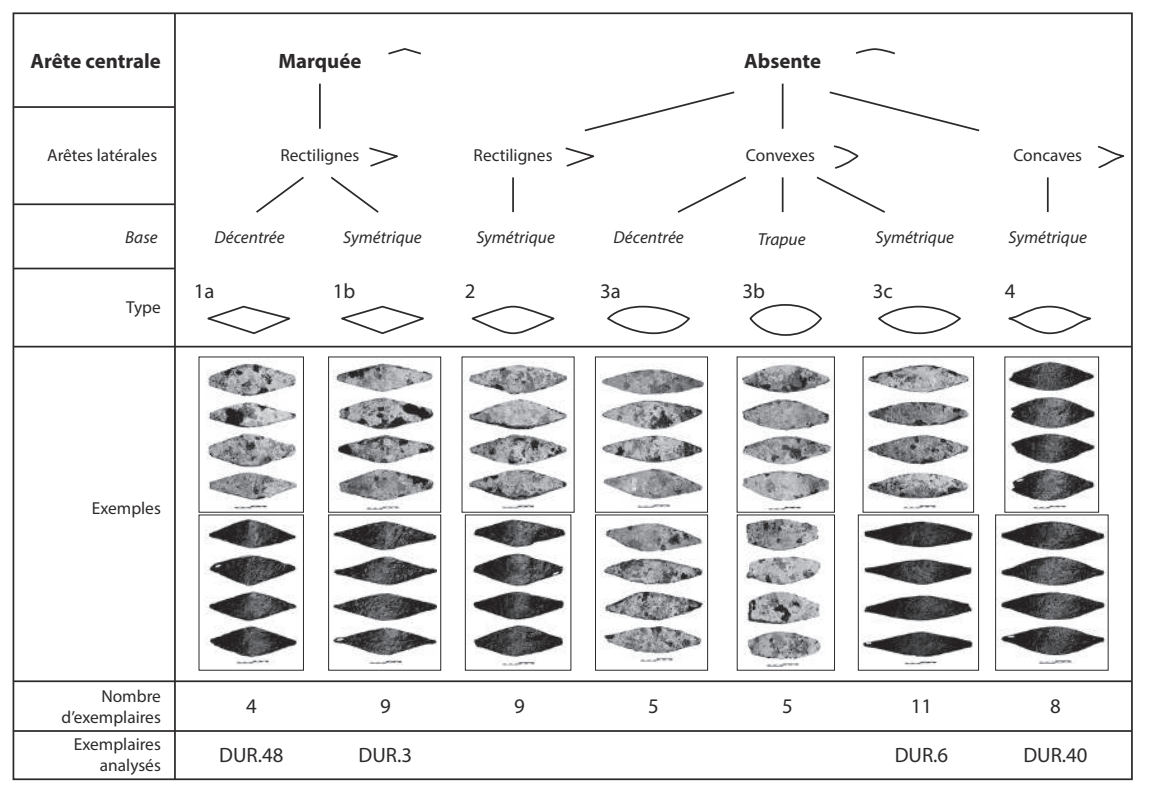

Figure 3: (Voir planche couleur VI) Classement typomorphologique des demiproduits en fer du dépôt de Durrenentzen. Les deux premiers niveaux de description (arêtes centrales et arêtes latérales) semblent être liés à des différences de techniques de finition en lien avec des " traditions techniques ». Le troisième niveau (base symétrique, décentrée, trapue) apparait comme une variabilité de mise en forme non mầtrisée.

Figure 3: (See colour plate VI) Typomorphological classification of the iron semi-products from the Durrenentzen deposit. The first two levels of description (central edges and lateral edges) seem to be linked to distinct finishing techniques in connection with "technical traditions". The third level (symmetric, off-center or thickset base) looks like a nonmastered shaping variability. 
Le protocole d'étude (Fluzin, 2002) repose en premier lieu sur une étude morpho-métrologique permettant de sélectionner l'axe de prélèvement. Après prises de vues macrographiques, les objets ont été tronçonnés longitudinalement sur la face la plus large afin d'étudier une section la plus étendue possible. Le tronçonnage a été effectué par l'entreprise Crelier SA à Belfort, au jet d'eau afin d'éviter toute altération structurale par échauffement. Les sections obtenues sont dégrossies au papier $\mathrm{SiC}$ (grades 80 à 1200) et polies à la pâte diamantée 6,3 et $1 \mu \mathrm{m}$. Elles sont ensuite observées intégralement au microscope métallographique avant et après attaques chimiques (nital $3 \%$ et Oberhoffer). Un repolissage est nécessaire entre les 2 attaques. Les zones représentatives de l'échantillon sont enregistrées à partir de prises de vue micrographiques, et les sections dans leur ensemble font l'objet de clichés macrographiques ou d'assemblages de microphotographies (cartographie).

Un logiciel de traitement d'image (LAS $\left.{ }^{\circledR}\right)$ permet, en dernier lieu, de quantifier la proportion surfacique des différents constituants (inclusions et porosités notamment) sur les faces polies de chaque objet. L'erreur relative d'estimation des surfaces est de l'ordre de $2 \%$.

\section{Analyses chimiques inclusionnaires}

Les analyses chimiques réalisées sur les inclusions de scorie ont pour but de définir leur signature chimique afin d'appréhender la provenance de l'objet et de caractériser la manière dont ce dépôt a pu être constitué. Il est possible d'aborder ces questions en comparant la composition des inclusions des demi-produits entre eux, mais également de comparer cette signature chimique à celle de scories de réduction issues de différents espaces de production potentiels (Coustures et al., 2003; Desaulty et al., 2009; Leroy et al., 2012a; Leroy et al., 2014; Coustures et al., 2014; Disser et al., 2015; Disser et al., 2016). Dans cet article seul le premier type de comparaison sera réalisé.

Le protocole d'analyse chimique se décline en deux phases. La première étape consiste à caractériser les éléments majeurs composant les inclusions d'un objet par spectrométrie EDS (pour Energy Dispersive Spectrometry) couplée au Microscope Electronique à Balayage (MEB-EDS). Cette étape permet notamment de distinguer les inclusions de réduction (portant la signature chimique de l'espace de production) de celles ajoutées au cours des phases de post-réduction (forge) (Dillmann et l'Héritier, 2007; Galili et al., 2015; Disser et al., 2014). La seconde étape porte sur le dosage de la composition en éléments traces composant les inclusions caractéristiques de la réduction par LA-ICP-MS (pour Laser Ablation-Inductively Coupled Plasma Mass Spectrometry). Le plus grand nombre d'éléments dosés permet alors d'aborder la question de la provenance de l'objet par comparaison des signatures chimiques (Desaulty et al., 2009; Leroy et al., 2012a; Disser et al., 2016).

La composition en éléments majeurs des inclusions est déterminée sur la coupe transversale étudiée en métallographie et métallisée (dépôt de carbone), par spectrométrie EDS (Silicon Drift Detector) couplé à un microscope électronique à balayage (modèle JEOL 7001-F).

La méthode consiste en l'acquisition de cartographies spectrales avec le logiciel d'imagerie MaxView (de la société SAMx) d'une taille de $512 \times 384 \mu \mathrm{m}$ avec une tension d'accélération de $15 \mathrm{kV}$, un courant de sonde à $20 \mathrm{nA}$, une constante de temps de $4 \mu$ s ( 80000 coups/s) et une résolution spectrale de $130 \mathrm{eV}$ (raie Ka du Mn). Ces cartographies permettent d'obtenir un spectre EDS pour chaque pixel de la zone cartographiée (le temps de comptage par pixel a été fixé à $18 \mathrm{~ms}$ ). Le logiciel permet de programmer l'acquisition de plusieurs cartographies spectrales grâce à la motorisation de la platine porte-échantillons (la durée d'acquisition d'une cartographie spectrale est d'environ $1 \mathrm{~h} 30$ ). Il est crucial d'étudier une surface représentative de l'objet. Pour ce faire, entre 3 et 10 cartographies spectrales sont programmées pour chaque échantillon, en fonction de la densité des inclusions, de leur taille, ainsi que de la taille de la coupe transversale. Après la séquence d'acquisition, afin d'extraire les inclusions de l'ensemble de la zone analysée, la cartographie est reconstituée à partir de la raie d'émission Ka de l'oxygène. Cela permet de séparer les inclusions riches en oxygène du métal qui en est dépourvu. Une procédure d'analyse d'image permet ensuite de recomposer un spectre-somme issu de la somme des spectres EDS associés à tous les pixels définissant la surface de chaque inclusion.

La surface minimale d'inclusion a été fixée à $50 \mu \mathrm{m}^{2}$ en raison de la difficulté de traiter un spectre-somme dont le rapport signal/bruit est trop faible. Le traitement des spectres est réalisé avec le logiciel IDFix. Chaque spectre est ensuite traité en suivant la méthode de quantification $\operatorname{Phi}(\rho z)$. Les pourcentages massiques pour chaque teneur considérée sont exprimés en équivalents oxydes et normalisés à $100 \%$. Les éléments dosés sont $\mathrm{Na}_{2} \mathrm{O}, \mathrm{MgO}, \mathrm{Al}_{2} \mathrm{O}_{3}, \mathrm{SiO}_{2}$, $\mathrm{P}_{2} \mathrm{O}_{5}, \mathrm{SO}_{3}, \mathrm{~K}_{2} \mathrm{O}, \mathrm{CaO}, \mathrm{TiO}_{2}, \mathrm{~V}_{2} \mathrm{O}_{5}, \mathrm{MnO}$ et $\mathrm{FeO}$. Pour les éléments dits mineurs de concentrations situées entre $0,5 \%$ (limite de détection) et $1 \%$ massique, l'erreur relative de quantification s'élève à $10 \%$. Pour les autres éléments (teneur supérieure à $1 \%$ massique) l'erreur relative est évaluée à $2 \%$. Les inclusions contenant du chlore n'ont pas été considérées, car cet élément est révélateur d'une corrosion aqueuse de l'objet postérieure à son enfouissement dans le 
sol, et que ces processus de corrosion sont susceptibles de modifier la composition des inclusions.

Lors de la transformation du minerai en métal, certains oxydes (adoptant un comportement lithophile) ne sont pas réduits et forment la scorie dont une partie reste piégée au sein du métal sous la forme d'inclusions. Ces oxydes sont principalement $\mathrm{MgO}, \mathrm{Al}_{2} \mathrm{O}, \mathrm{SiO}_{2}, \mathrm{~K}_{2} \mathrm{O}$ et $\mathrm{CaO}$. Ils ont été nommés NRC (pour Non Reduced Compounds - Dillmann et l'Héritier, 2007). Ils constituent ainsi la signature d'un système de réduction incluant le minerai, les cendres du charbon, la contribution de l'argile des parois de four et d'éventuels ajouts (Dillmann et L'Héritier, 2007). Toutefois, pendant l'étape d'épuration du métal, destinée à transformer la masse brute en demi-produit, de même que lors d'opérations de forge plus avancées dans la chaîne opératoire, l'usage d'ajouts peut être nécessaire afin de réaliser des soudures ou d'éviter que le métal ne s'oxyde. Certaines inclusions présentes dans le métal peuvent ainsi être entièrement ou partiellement issues des ajouts de forge et ne résultent pas ou plus uniquement de la réduction du minerai. Il est ainsi indispensable de vérifier que les inclusions prises en compte dans l'étude sont bien issues de l'étape de réduction et de discriminer celles formées ou polluées lors du forgeage. Dans un premier temps, l'étude métallographique permet de localiser, au sein de l'objet, les éventuelles lignes de soudures contenant des inclusions formées par les ajouts. Par la suite, l'analyse de la composition en éléments majeurs NRC permet de les caractériser et de les différencier des autres inclusions non-polluées. Il est également possible, lorsqu'aucune soudure n'est visible en métallographie, de caractériser chimiquement un très grand nombre d'inclusions et, à travers l'emploi de méthodes statistiques, de discriminer les groupes d'inclusions dont la signature n'est pas issue du minerai (Disser et al., 2014). Ces procédures ont été suivies pour chacune des sections polies analysées et ont permis de distinguer les familles d'inclusions correspondant à l'étape de réduction. Dans la suite de cet article, seules ces dernières seront considérées.

Pour chacun des objets analysés, une dizaine d'inclusions représentatives de la réduction a ensuite fait l'objet d'une étude par LA-ICP-MS réalisée au Centre Ernest-Babelon (IRAMAT, Orléans). L'appareillage utilisé est un VG Plasma Quad PQXS couplé à un laser du type Nd:YAG à fréquence quadruplée de longueur d'onde de $266 \mathrm{~nm}$. Le laser, dont le diamètre peut être ajusté à l'aide d'un ensemble de collimateurs et d'objectifs ( $40-200 \mu \mathrm{m})$, est dévié vers l'échantillon à l'aide d'un miroir incliné à $45^{\circ}$. La taille de l'ablation est généralement du même ordre de grandeur que celle de l'inclusion, ou suffisamment représentative par rapport à l'échelle des phases constituant l'inclusion.
L'analyse est ainsi "globale » et le volume du prélèvement permet d'obtenir des données représentatives de l'inclusion dans son ensemble. La quantité de matière prélevée et, par conséquent, le nombre d'ions détectés instantanément sont dépendants de la fréquence du laser. Les paramètres déterminés de façon à obtenir un signal suffisant durant l'acquisition sont les suivants : réglage de la fréquence du laser entre 5 et 7 hertz et temps d'ablation fixé à 50 secondes. Au cours de l'ablation, un flux d'argon balaie l'intérieur de la cellule et entraîne les particules prélevées jusqu’à la torche à plasma où elles sont atomisées et ionisées. La détection des ions s'effectue à l'aide d'un multiplicateur d'électrons secondaires de type Channeltron à dynode continue. Comme il n'existe aucune garantie de reproductibilité de l'ablation et du transfert de la matière prélevée d'une ablation à l'autre, il est nécessaire d'utiliser un étalon interne (28Si) qui aura subi les mêmes phénomènes au cours de l'analyse, et à l'aide duquel chaque mesure pourra être corrigée. La procédure de calcul des concentrations fait également intervenir la composition connue et certifiée du verre de référence NISTSRM610. Au total, 39 éléments chimiques incluant la série des Terres Rares sont mesurés pendant l'analyse. En fonction de la taille de l'inclusion et de sa teneur en $\mathrm{Si}$, et dans les conditions expérimentales adoptées, les limites de détection atteintes varient entre quelques ppb et $15 \mathrm{ppm}$ pour tous les éléments à l'exception du Ti (lod = 130 ppm). L'erreur relative de la mesure pour les éléments traces est comprise entre 5 et $10 \%$.

Pour les compositions en éléments traces, avant exploitation par des approches statistiques, un traitement spécifique des données est réalisé pour normaliser les teneurs. C'est la normalisation en rapports logarithmiques qui a été privilégiée suivant la procédure proposée par Leroy et Disser pour les études inclusionnaires (Leroy et al., 2012a; Disser et al., 2014) car elle présente l'avantage de s'affranchir des effets d'enrichissement dus à l'étape de réduction (diminution de la proportion de fer dans la scorie par rapport à la proportion de fer dans le minerai). Pour chaque élément est calculée une valeur $X_{E}$ qui sera utilisée ensuite :

$X_{E}=\log ([E])-\frac{1}{N} \sum_{K=1}^{N} \log \left(\left[E_{K}\right]\right)$

où les $\left(E_{K}\right)$ sont les $\mathrm{N}$ éléments les moins variables et les mieux mesurés. L'utilisation de l'échelle logarithmique permet d'obtenir des valeurs et des erreurs absolues comparables pour les différents éléments, même si initialement ils présentent des teneurs mesurées très différentes. On parle alors de mise à l'échelle des données (réduction). Ainsi, chaque 
échantillon analysé sera caractérisé par une observation, ellemême définie par l'ensemble des $X_{E}$ de chacun des éléments chimiques sélectionnés pour l'étude de provenance. Dans notre cas d'étude, les éléments pris en compte lors de l'analyse statistique sont: Ce, Eu, Gd, La, Nd, Pr, Sm, Th, U et Y. Les éléments $E_{K}$ sont : Ce, Eu, La, Sm et Y.

La répartition de ces observations $\mathrm{X}_{E}$ dans l'espace multivarié a ensuite été étudiée afin de caractériser les relations des données entre elles, et donc de remonter à des indices de provenance : origine différente ou commune (Leroy et al., 2014; Disser et al., 2016). Deux analyses multivariées couramment utilisées dans le domaine de l'archéologie ont été employées; l'analyse en composantes principales (ACP), basée sur une matrice composée des coefficients de corrélation de Pearson, et la classification ascendante hiérarchique (CAH), par dissimilarité en fonction des distances euclidiennes par la méthode d'agrégation de Ward. Les traitements statistiques ont été réalisés à partir du logiciel XLSTAT Version 2016.01.26779 de la société ADDINSOFT.

\section{Datations par le radiocarbone}

Les quatre demi-produits ont été datés par dosage du radiocarbone. Au cours de la chaîne opératoire sidérurgique ancienne, du charbon de bois est utilisé pour la réduction en métal des oxydes contenus dans le minerai. Lors de cette transformation, une partie du carbone du charbon de bois peut s'associer au fer sous la forme de cémentite dans les zones aciérées du métal produit. Ainsi, l'extraction du carbone du métal et la détermination de ses rapports isotopiques permettent de dater le bois qui a servi à obtenir le charbon de bois.

Les zones aciérées (et donc contenant le carbone à doser) sont repérées lors de l'étude métallographique de l'objet (voir supra). L'attaque à l'acide nitrique employé pour cette étude assure également le nettoyage de la surface exposée en décapant la couche externe, éliminant ainsi toute source de pollution carbonée éventuellement apportée par la coupe de l'objet ou le polissage. Cette approche permet de directement sélectionner, sur les coupes métallographiques transversales, les zones les plus carburées et de les prélever localement en utilisant des forets de diamètre millimétrique $(\varnothing 2 \mathrm{~mm}$, $\varnothing 3,5 \mathrm{~mm}, \varnothing 4 \mathrm{~mm}$ ). Nous avons utilisé deux types de forets : un foret en acier avec un revêtement TiN (Nitrure de Titane) ou avec un revêtement CoB (Cobalt-Bore), afin d'éviter toute pollution en carbone. Cette approche, concentrant les prélèvements sur les zones les plus aciérées de l'objet, permet d'étudier des objets de teneur moyenne relativement faible mais contenant des zones de plus forte concentration. Prélever sur coupe transversale garantit aussi l'élimination des produits de corrosion et des contaminations du milieu dans lequel a séjourné l'échantillon.

Le carbone présent dans les prélèvements est ensuite extrait par combustion. Entre 50 et $300 \mathrm{mg}$ de métal sont scellés dans un tube en quartz avec un excès d'oxyde de cuivre $(\mathrm{CuO})$ en grains jouant le rôle d'oxydant et un fil d'argent $(\mathrm{Ag})$ destiné à piéger des éléments tels que les halogènes, susceptibles de perturber la réaction suivante de graphitisation (transformation $\mathrm{du} \mathrm{CO}_{2}$ en graphite pur). Le $\mathrm{CuO}$ et l'Ag sont préalablement débarrassés de toute trace de carbone en étant placés sous flux d'oxygène, pendant 4 heures à $815^{\circ} \mathrm{C}$ avant utilisation. Le tube contenant l'échantillon est porté à $850^{\circ} \mathrm{C}$ pendant 5 heures et le $\mathrm{CO}_{2}$ produit est collecté dans une ampoule en verre après avoir été déshydraté sur un piège carboglace/alcool à $-80^{\circ} \mathrm{C}$. L'efficacité de l'extraction du carbone en fonction de l'excès d'oxygène fourni par le $\mathrm{CuO}$ dans le tube a été testée sur des aciers et des échantillons de fonte (de 0,2\% à $3 \% \mathrm{C}$ ). En effet, certains prélèvements étant plus pauvres en carbone ou de faible masse, il est indispensable que l'étape de combustion permette d'extraire le plus complètement possible le carbone présent dans l'échantillon. Les résultats ont montré que l'extraction du carbone était complète lorsque la combustion des échantillons était réalisée avec un excès d'oxygène tel que $\mathrm{CuO} / \mathrm{Fe}=5$ (Leroy et al., 2015a). Finalement, les échantillons de $\mathrm{CO}_{2}$ sont réduits en graphite et mesurés par Spectrométrie de Masse par Accélérateur (SMA) au LMC14. Les dates radiocarbones obtenues sont transformées en âges calendaires au moyen du logiciel Oxcal 4.2.4 (Ramsey et Lee, 2013) qui utilise la courbe de calibration IntCal 13 (Reimer et al., 2013). Deux mesures radiocarbones ont été obtenues à l'échelle de chaque masse métallique datée afin de vérifier tout effet " vieux bois » potentiel. Ces deux mesures radiocarbones étant associées à la même masse métallique, elles datent donc le même évènement (l'âge de la masse). Nous avons choisi de les combiner en utilisant l'outil R-Combine dans Oxcal afin d'obtenir une densité d'âge par demi-produit.

\section{Résultats}

\section{Caractéristiques métallographiques}

\section{Qualité inclusionnaire}

Le premier critère de caractérisation est celui de la qualité inclusionnaire (figure 7). Il s'agit de déterminer la proportion des inclusions présentes au sein de la matrice métallique par leur teneur surfacique sur les sections polies, mais aussi de déterminer leur nature et leur morphologie. On note une grande variabilité au sein du corpus. Les inclusions (porosités et inclusions silicatées) occupent ainsi entre 3 et $17 \%$ des 
surfaces étudiées, en fonction des exemplaires (tableau 1). Deux demi-produits se caractérisent par une propreté inclusionnaire évaluée comme excellente (entre 0 et $5 \%$ ) d'après le référentiel mis en place sur des objets de la même période (Bauvais et Fluzin, 2006). Un autre montre une propreté évaluée comme bonne (entre 5 et $10 \%$ ), et un dernier une qualité appréciée comme mauvaise (entre 15 et $25 \%$ ).

La répartition des inclusions s'avère également variable. Pour DUR.40 (figure 5), elles sont plus fréquentes en périphérie de la section étudiée, alors que pour DUR.3 et DUR.48 (figure 4), elles se concentrent principalement en partie centrale. Dans cette zone, elles présentent le plus souvent des contours déchiquetés car elles n'ont été que peu déformées par le martelage.

Les porosités sont plus nombreuses pour DUR.6, DUR.3 et DUR.48, ce qui témoigne d'un processus de compactage sommaire. Les porosités formées durant le processus de réduction n'ont pas encore été suffisamment écrasées sur elles-mêmes durant l'étape d'épuration-compactage. Dans l'exemplaire DUR.48 le métal est d'ailleurs mêlé localement à une forte proportion de petites inclusions de scories, témoignant également d'une agglomération incomplète de la matrice métallique.

Les inclusions présentes au sein de tous les objets étudiés sont multiphasées et constituées de silicates de fer de type fayalite $\left(\mathrm{Fe}_{2} \mathrm{SiO}_{4}\right)$, de phases vitreuses et de dendrites de wüstite $(\mathrm{FeO})$, en proportions variables. Elles peuvent également s'avérer être totalement amorphes et siliceuses. DUR.40 et DUR.3 contiennent également des inclusions constituées quasi exclusivement de globules de wüstite liés par une matrice silicatée. Leur formation est due à l'insertion d'oxydes de fer issus de l'oxydation du métal (battitures) durant les opérations de forgeage à chaud. On note l'absence, sur toutes les surfaces des demi-produits, de battitures ou de nappage de scorie périphérique issu des opérations de forge.

\section{Composition des alliages}

La composition de l'alliage fer/carbone et la présence de phosphore ont été étudiées grâce aux attaques chimiques aux réactifs Nital $3 \%$ et Oberhoffer réalisées après polissage (figure 7). Elles révèlent également une certaine disparité entre les exemplaires (tableau 1).

DUR.6 est un produit ferritique (la ferrite occupe $74 \%$ des surfaces étudiées), localement aciéré autour de $0,4 \% \mathrm{C}$, au cœur de l'objet. Des structures dites " fantômes " résultant d'hétérogénéités de répartition du phosphore dans la ferrite ont été observées de manière locale (Vega et al., 2002). DUR.40 et DUR.48 sont constitués de fer phosphoreux. Les zones de ferrite occupent respectivement 90 et $60 \%$ des surfaces étudiées et comprennent des structures " fantômes » visibles sur des surfaces étendues des sections étudiées.

Le dernier exemplaire, DUR.3, est quant à lui un produit majoritairement aciéré, sous la forme de zones ferrito-perlitiques à $0,2 / 0,4 \% \mathrm{C}$, qui occupent $45 \%$ de la surface observée, et dans lesquels la perlite est de forme lamellaire. Sur près d'un cinquième de la surface, la teneur en carbone atteint $0,8 \%$. Dans les zones de ferrite, qui occupent environ $35 \%$ de la surface de la section étudiée, les structures " fantômes " n'ont été observées que de manière très ponctuelle.

Au sein des quatre demi-produits, les zones les plus carburées se localisent autour des plus larges porosités favorisant l'accès du CO gazeux lors de la réduction, permettant une cémentation de la matrice métallique par diffusion du carbone.

\section{Techniques de fabrication}

Deux chaînes opératoires de fabrication peuvent être distinguées (figure 7). Une première technique concerne DUR.3 et DUR.6 (figure 4). Ces demi-produits ont été mis en forme par écrasement simple de la masse de métal sur elle-même. Les extrémités et surfaces comprennent un grand nombre de soudures de faible étendue, débouchant en surface. Elles résultent très probablement de la soudure sur elles-mêmes d'excroissances métalliques présentes sur la masse brute de métal initiale. Cette dernière devait ainsi être constituée de parties métalliques filamentaires ou partiellement indépendantes en périphérie.

\begin{tabular}{|c|c|c|c|c|c|c|}
\hline \multirow{2}{*}{$\begin{array}{c}\text { Demi- } \\
\text { produit }\end{array}$} & \multirow{2}{*}{ inclusions } & \multicolumn{3}{|c|}{ Alliage fer/carbone } & Structures fantômes \\
de phosphore & $\begin{array}{c}\text { Nombre de masses } \\
\text { de métal } \\
\text { ferrite à } 0,2 \% \mathrm{C} \\
\text { majoritaire }\end{array}$ & 0,2 à $0,6 \% \mathrm{C}$ & 0,7 à $0,8 \% \mathrm{C}$ & $19 \%$ & Locales & 1 \\
\hline DUR.3 & $17 \%$ & $36 \%$ & $45 \%$ & $6 \%$ & Locales & 1 \\
\hline DUR.6 & $3 \%$ & $74 \%$ & $20 \%$ & $4 \%$ & Étendues & 2 \\
\hline DUR.40 & $4 \%$ & $90 \%$ & $6 \%$ & $24 \%$ & Étendues & 2 \\
\hline DUR.48 & $7 \%$ & $66 \%$ & $10 \%$ & & 2 \\
\hline
\end{tabular}

Tableau 1 : Synthèse des caractéristiques métallographiques des demi-produits en fer étudiés.

Table 1: Synthesis of the metallographic characteristics of the studied iron semi-products. 

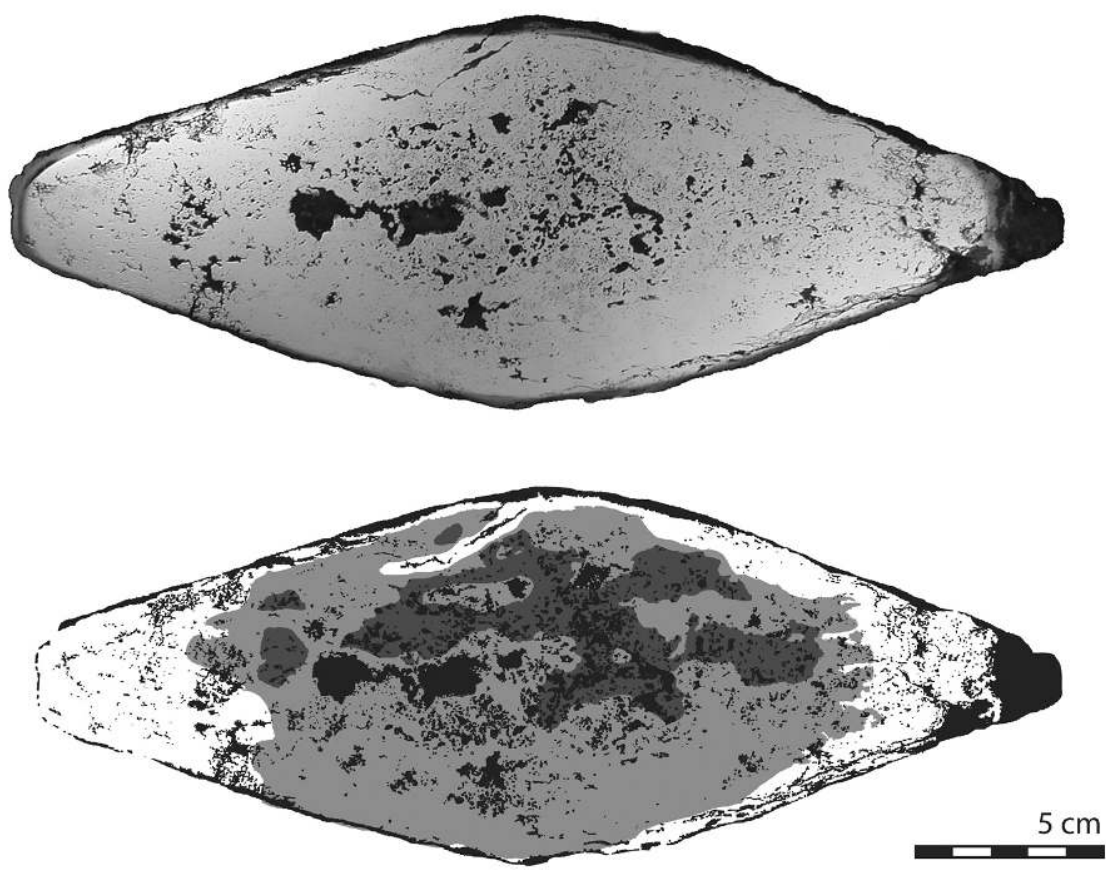

Inclusions : porosités, scorie et produits de corrosion

Ferrite à acier à $0,2 \% \mathrm{C}$ majoritaire

Acier de 0,2 à $0,6 \%$ C majoritaire

Acier de 0,7 à $0,8 \%$ C majoritaire

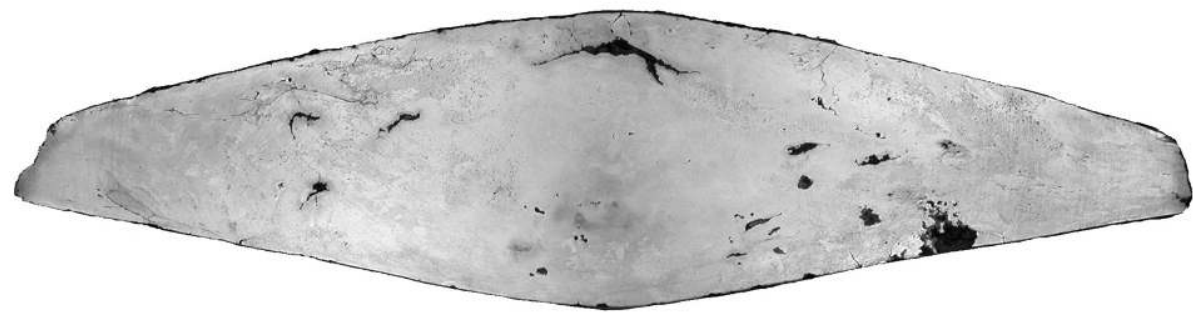

Figure 4 : Sections analysées de DUR.3 (en haut) et DUR.6 (en bas). Figure 4: Analyzed sections of DUR.3 (up) and DUR.6 (down).

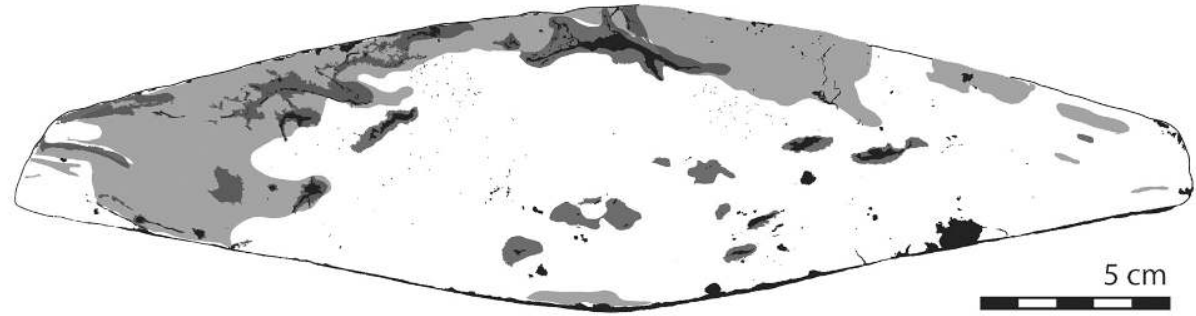

Au sein de DUR.40 et DUR.48 des soudures étendues ont été observées, elles traversent de part en part les sections étudiées et témoignent de l'assemblage de deux masses métalliques. La section étudiée pour DUR.40 coupe la soudure perpendiculairement (figure 5). Un liseré d'inclusions constituées majoritairement de très gros globules de wüstite la souligne. Ces dernières se sont très probablement formées par l'oxydation locale du métal au moment de la soudure dans une zone du foyer à l'atmosphère oxydante. Localement, les surfaces des deux masses métalliques ont même dépassé le point de fusion de la ferrite $\left(1536^{\circ} \mathrm{C}\right)$. On parle alors de "brûlure du métal ». La section de DUR.48 a, quant à elle, coupé la soudure presque parallèlement à son plan (figure 6). Elle est de moins bonne facture que la pré- 


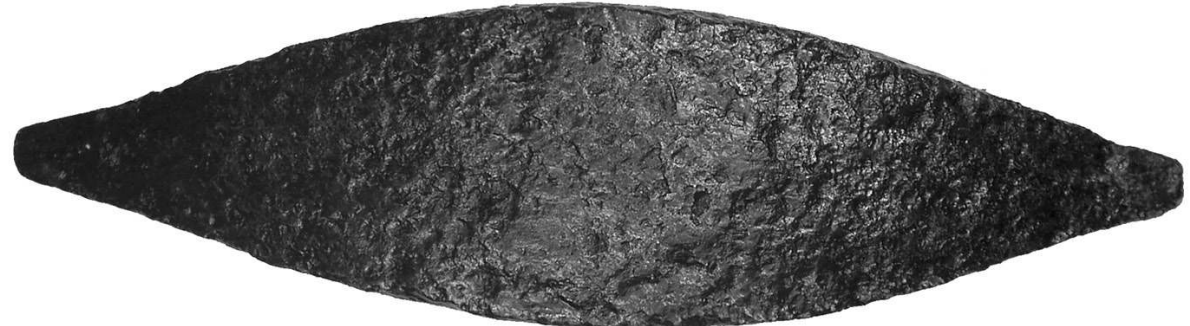

।
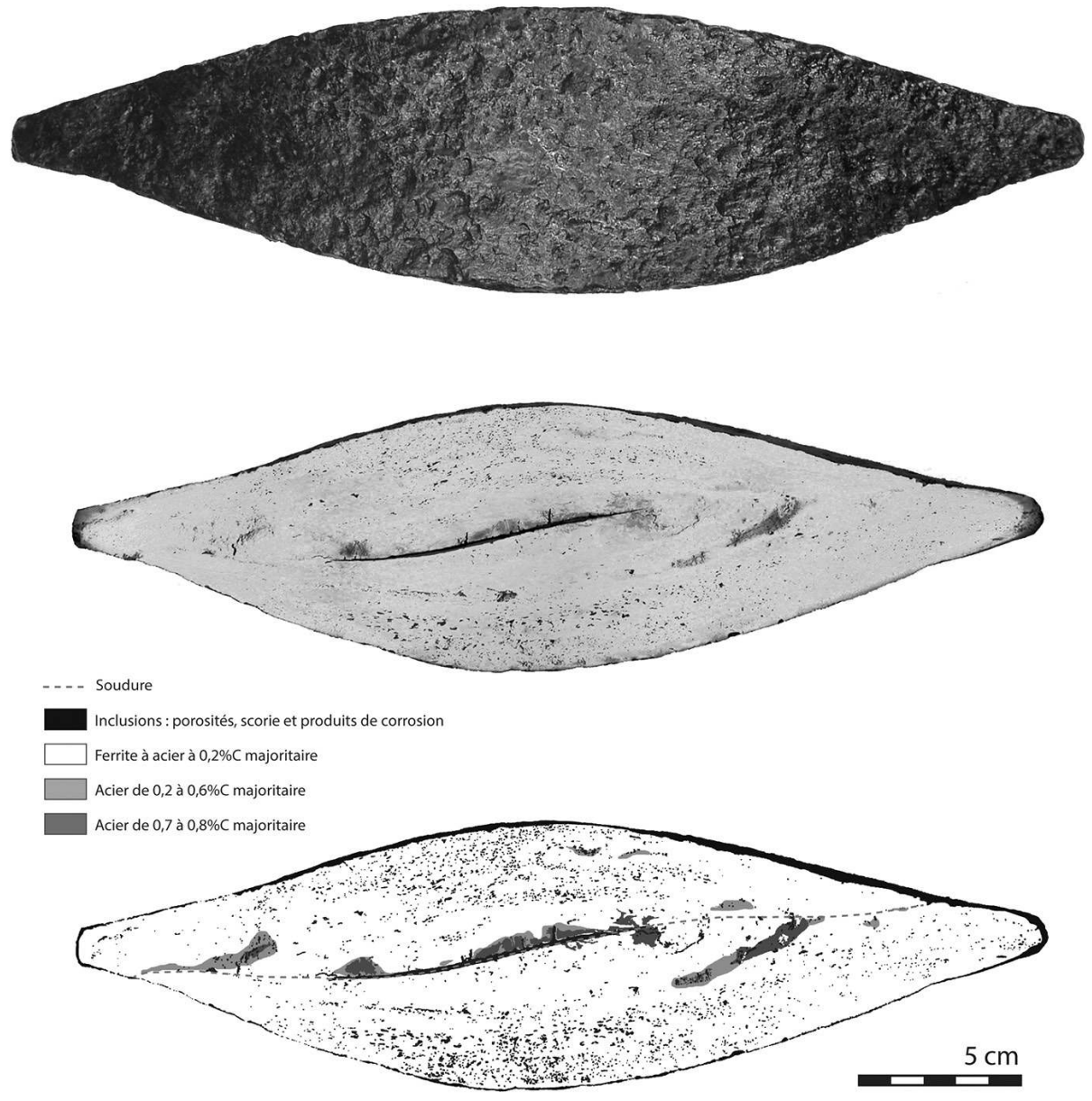

Figure 5 : Vues macroscopiques et section analysée de DUR.40.

Figure 5: Macroscopic view and analyzed section of DUR. 40 . cédente. Certaines zones sont parfaitement jointives tandis que d'autres comprennent un liseré d'oxydes interstitiel, ou sont disjointes.

Les soudures n'ont pu être observées qu'en fonction d'un seul plan, longitudinal. Il n'est donc pas possible de déterminer si les deux masses de métal sont totalement distinctes en trois dimensions ou correspondent au repli sur elle-même d'une unique masse métallique. L'analyse chimique inclusionnaire permettra de préciser cet aspect.

\section{Synthèse par demi-produit}

DUR.3 est un demi-produit mis en forme par déformation simple d'une unique masse de métal (figure 4). D’une qualité d'épuration médiocre, son taux de compacité reste limité (17\% d'inclusions subsistantes). Des porosités de taille centimétrique sont ainsi encore visibles en partie centrale de la section étudiée. Il est possible que la déformation de ce produit massif et principalement constitué d'acier n'ait pas été aisée, ce qui expliquerait que l'étape d'épurationcompactage ait été interrompue avant son achèvement, après avoir abouti à un produit d'apparence régulière. 
Figure 6 : Vues macroscopiques et section analysée de DUR.48.

Figure 6: Macroscopic view and analyzed section of DUR. 48.

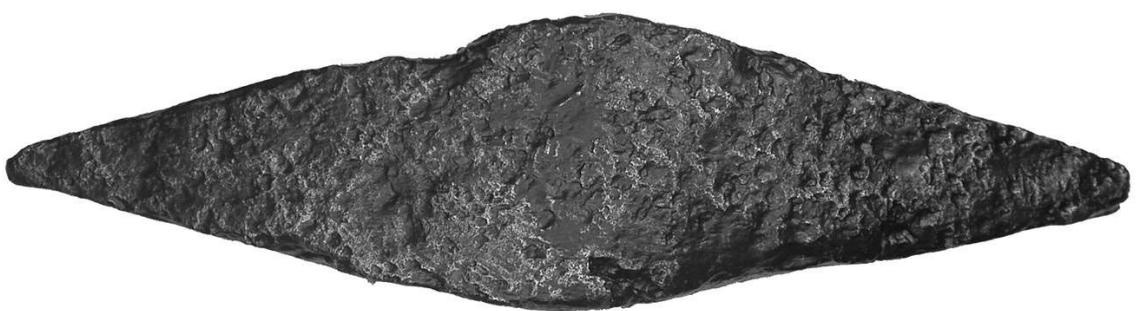

।
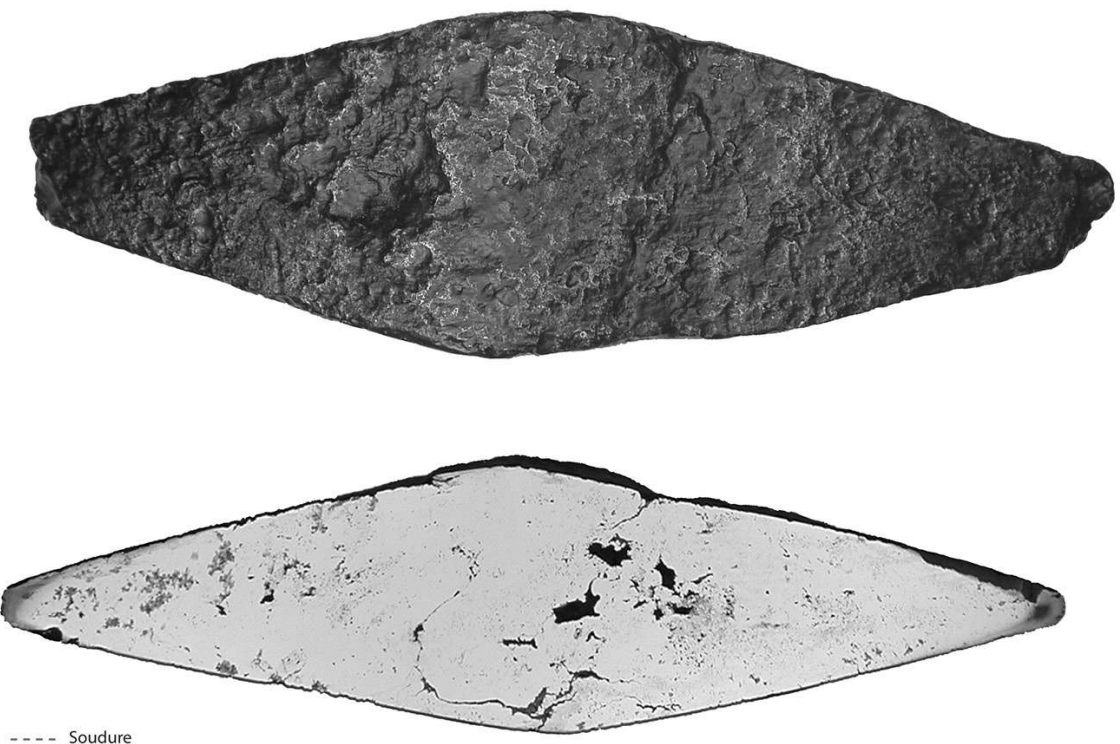

Inclusions : porosités, scorie et produits de corrosion

ferrite à acier à $0,2 \% \mathrm{C}$ majoritaire

Acier de 0,2 à $0,6 \%$ C majoritaire

Acier de 0,7 à $0,8 \% C$ majoritaire

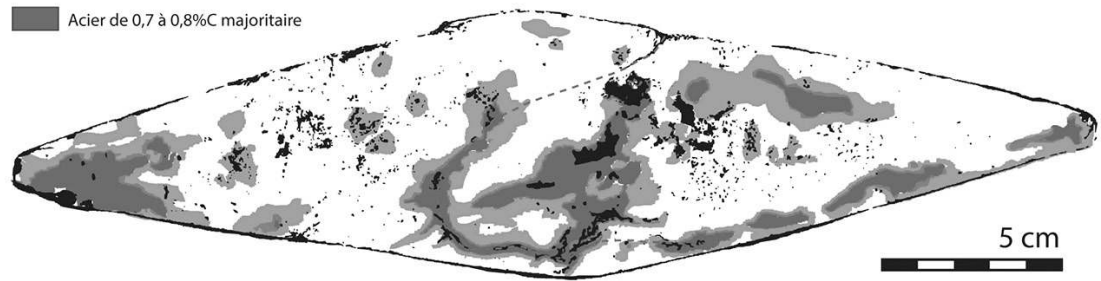

DUR.6 résulte également d'une mise en forme par simple déformation d'une unique masse de métal (figure 4). Il s'avère néanmoins bien plus compact, avec seulement $3 \%$ d'inclusions non métalliques encore présentes. Produit ferritique comprenant localement du phosphore, d'après l'observation de structures fantômes présentes en proportions peu étendues, il s'avère également plus malléable et peut être plus facile à compacter. Il est également possible que la masse de métal initiale ait été déjà relativement dense.

DUR.40 procède de l'assemblage de deux masses de métal constituées chacune de fer phosphoreux (figure 5). La présence de ce composé devait engendrer des contraintes particulières en forge : déformation à des températures plus faibles (Vega et al., 2002), mais aussi moindre malléabilité par rapport à la ferrite. La répartition des inclusions, situées préférentiellement en surface du demi-produit, laisse supposer une épuration préalable des deux masses de métal, avant leur soudure sur leur face la plus large. En résulte un demi-produit aux qualités homogènes et très bien épuré.

DUR. 48 présente des caractéristiques très proches de celles de DUR.40 (figure 6). Il est également constitué par l'assemblage de deux masses de métal. Toutes deux hétérogènes, elles sont majoritairement constituées de fer phosphoreux, avec des plages étendues d'acier. Il s'agit d'un produit pour lequel le travail d'épuration a été poussé. 


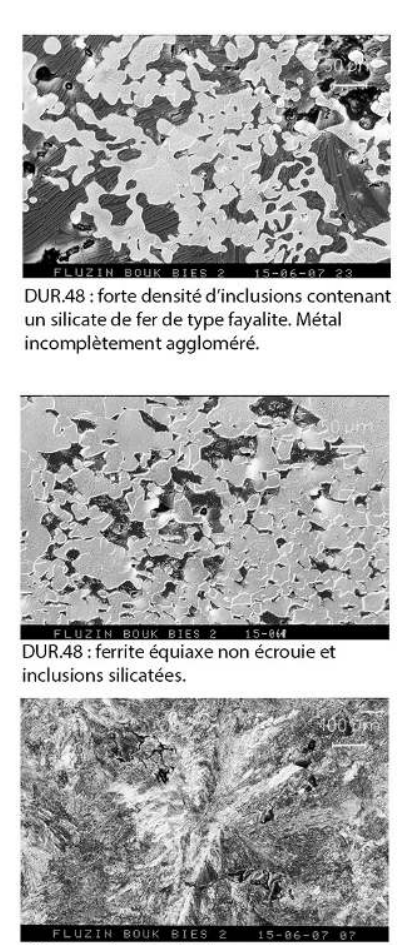

DUR. 48 : acier à $0,8 \%$ C (eutectoïde).

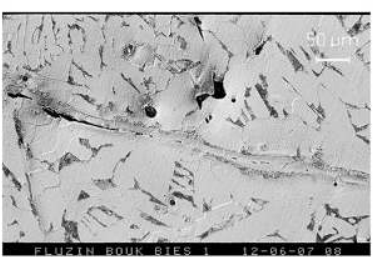

DUR.40 : soudure entre deux masses aciérées autour de $0,2 \%$ C parfaitement réalisée.

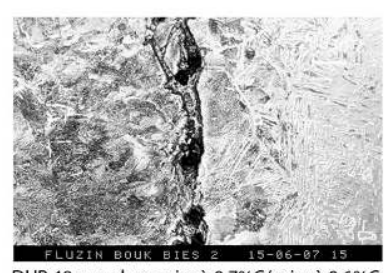

DUR.48: soudure acier à $0,7 \% \mathrm{C} /$ acier à $0,6 \% \mathrm{C}$.
Qualité inclusionnaire

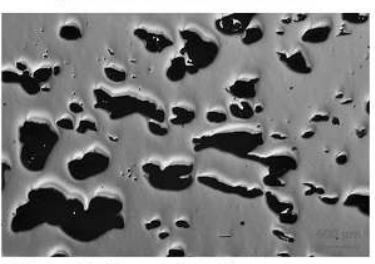

DUR. 3 : arrondis.

\section{Composition de l'alliage}

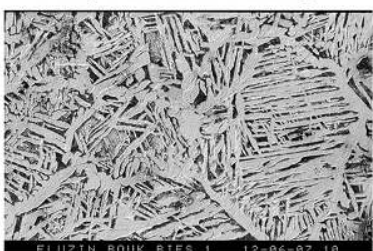

DUR.40 : acier à 0,3\% (ferrite aciculaire, indiquant une surchauffe locale).

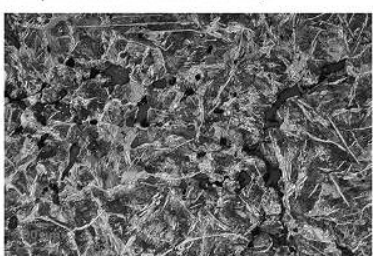

Techniques de fabrication

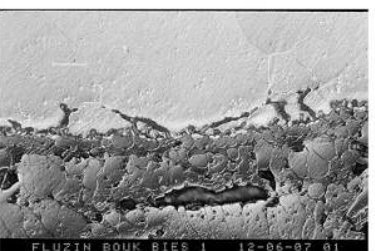

DUR.40 : décohésion locale de la ferrite, en li avec un liseré d'oxyd

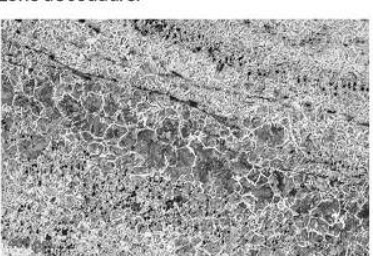

DUR.6: fibrage d'acier à différentes teneurs en carbone, résultant de l'écrasement d'un même masse de métal sur elle-même.

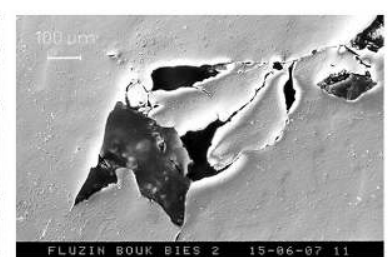

DUR.48 : inclusions majoritairement siliceuses.

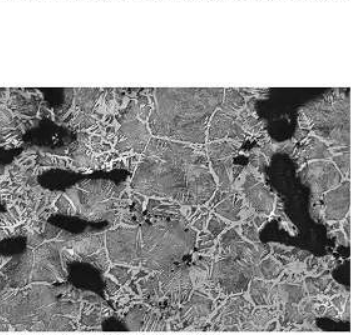

DUR.3 : acier autour de $0,5 \%$ C.

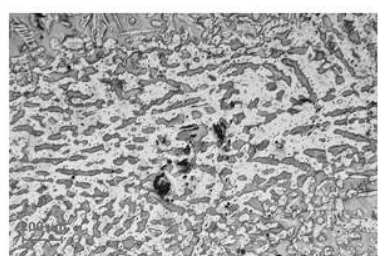

DUR.40 : structures fantômes témoignant d'anomalies de teneur en phosphore.

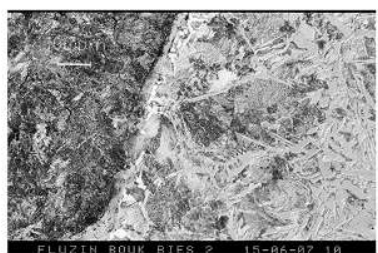

DUR.48 : soudure acier $0,8 \% \mathrm{C} / \mathrm{acier} 0,5 \% \mathrm{C}$ parfaitement réalisée.

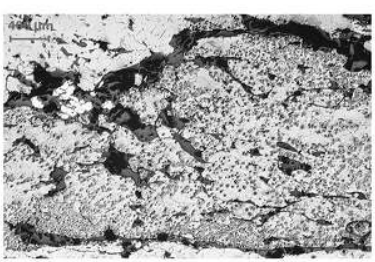

DUR 6 : soudure d'une massede métal écrasée sur elle-même.
Figure 7 : (Voir planche couleur VII) Clichés micrographiques illustrant la qualité inclusionnaire, la composition chimique et les techniques de fabrication mises en évidence lors de l'analyse métallographique des demi-produits en fer étudiés.

Figure 7: (See colour plate VII) Micrographic pictures illustrating inclusional quality, chemical composition and manufacturing techniques highlighted by the metallographic analyses of the studied iron semi-products.
Ainsi, malgré la grande homogénéité morphologique et métrologique du lot de demi-produits, l'étude de quatre exemplaires met en évidence une certaine variabilité dans la nature des masses de métal qui les constituent et, surtout, des savoir-faire de forgeron très différents dans les gestes techniques ayant abouti à leur réalisation. Ceci peut s'expliquer par la nature des masses initiales de métal brut utilisées dans la fabrication de ces demi-produits. Leurs différences de volumes, de composition et de compacité ont conduit à l'obtention de demi-produits aux qualités différenciées.
Soulignons cependant que la qualité d'épuration et la nature de l'alliage sont homogènes au sein d'un même demi-produit, même lorsqu'il est constitué de deux masses de métal. Enfin, les caractéristiques des demi-produits étudiés révèlent un grand nombre d'indices indiquant un travail peu poussé : inclusions nombreuses et/ou de grande taille, zones métalliques incomplètement agglomérées. Ainsi, dans la chaîne opératoire, ces objets sont bien plus proches du résultat de l'étape de réduction que de celle de l'élaboration finale d'objets. 


\section{Compositions chimiques des inclusions}

L'analyse inclusionnaire des quatre demi-produits a été menée en recherchant la plus grande représentativité des zones étudiées. Neuf cartographies EDS ont été réalisées au sein du demi-produit DUR.40, composé de deux masses métalliques soudées entre elles; cinq sur la première masse et quatre sur la seconde. Au total 815 inclusions ont été caractérisées. Pour le demi-produit DUR.48, également composé de deux masses, neuf cartographies (5 pour la première masse et 4 pour la seconde) représentent au total 1740 inclusions analysées. Les deux suivants (DUR. 3 et DUR.6), composés d'une seule masse, ont respectivement subi cinq et trois cartographies représentant 355 et 651 inclusions.

La figure 8 illustre les deux principaux rapports (\%mass $\mathrm{Al}_{2} \mathrm{O}_{3} / \%$ mass $\mathrm{SiO}_{2}$ et \%mass $\mathrm{K}_{2} \mathrm{O} / \%$ mass $\mathrm{CaO}$ ) caractérisant la composition des inclusions des demi-produits. Sur le demi-produit DUR.40, caractérisé par la soudure de deux masses de métal, il n'est pas possible de distinguer les inclusions de chacune des masses constitutives du demi-produit (figure 8). La très forte ressemblance chimique entre les deux parties du demi-produit laisse davantage envisager qu'il s'agit de deux fragments d'une même masse brute issue d'une unique réduction. Toutefois, dans l'éventualité d'une chaîne opératoire particulièrement bien maitrisée et conduite dans des conditions identiques, ces deux masses peuvent être issues de deux réductions du même atelier. Afin de comparer la composition chimique des inclusions plus avant, nous avons, pour chacune des masses étudiées, évalué une composition moyenne des inclusions calculée en pondérant la teneur de chaque inclusion par sa surface (Dillmann et L'Héritier, 2007), tel que :

$$
\% E^{*}=\sum_{i=1}^{n}\left(\% E_{i} \times \frac{S_{i}}{S_{T}}\right)
$$

où $\% E^{*}$ est la composition pondérée de l'élément ou de l'oxyde considéré, $\% E_{i}$ est le pourcentage massique de l'élément ou de l'oxyde contenu dans l'inclusion $i$, Si est la surface de l'inclusion analysée et $n$ le total du nombre d'inclusions. Ces teneurs pondérées seront par la suite indiquées par une ${ }^{*}(\% *)$.

Par ailleurs, en plus des $\mathrm{NRC}\left(\mathrm{SiO}_{2}, \mathrm{Al}_{2} \mathrm{O}_{3}, \mathrm{~K}_{2} \mathrm{O}\right.$ et $\left.\mathrm{CaO}\right)$ dont on peut comparer les rapports, nous avons également considéré les teneurs moyennes en $\mathrm{MnO}$ et $\mathrm{P}_{2} \mathrm{O}_{5}$. Ces deux composés n'étant pas strictement lithophiles, leur teneur dans les inclusions pourra dépendre plus fortement des conditions thermodynamiques locales dans le fourneau que celles des NRC. C'est la raison pour laquelle, afin de
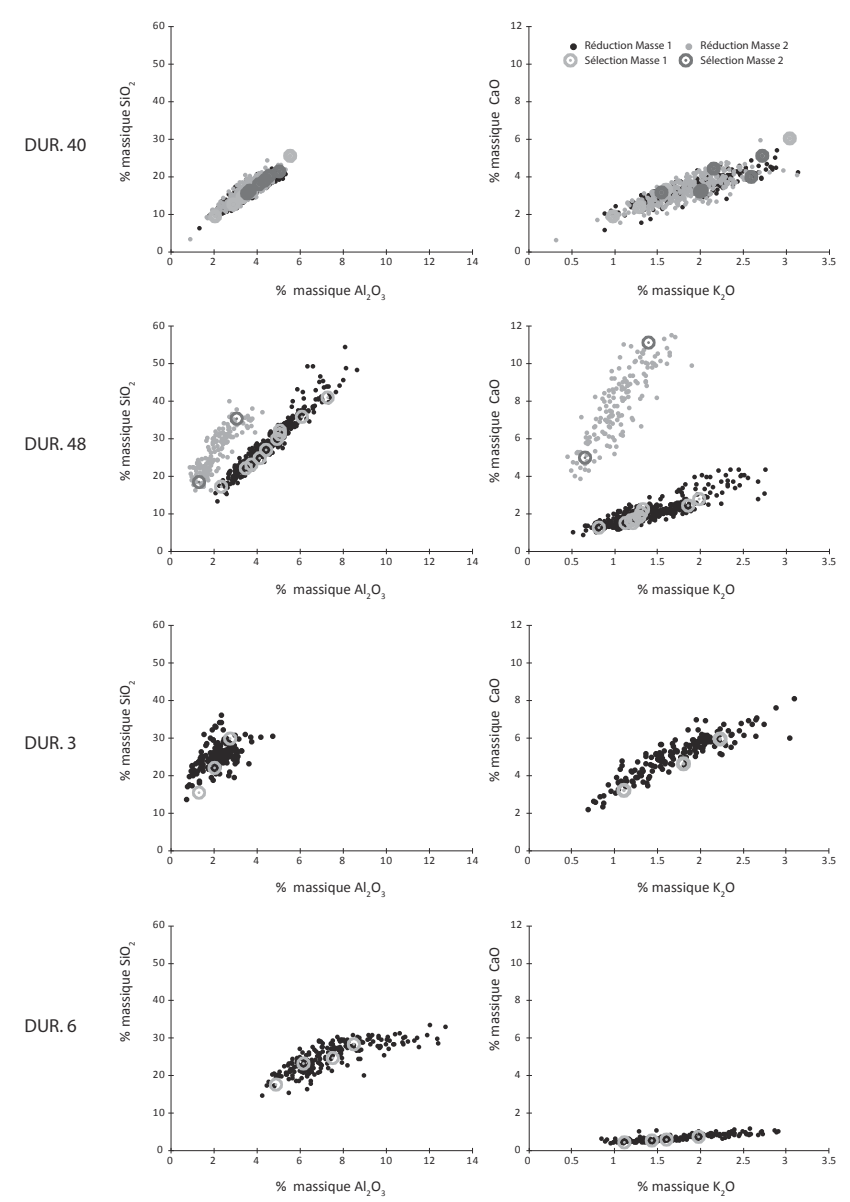

Figure 8 : (Voir planche couleur VIII) Rapports $\mathrm{Al}_{2} \mathrm{O}_{3} / \mathrm{SiO}_{2}$ et $\mathrm{K}_{2} \mathrm{O} / \mathrm{CaO}$ des 4 demi-produits analysés (exprimés en \%massique). Chaque point représente les teneurs obtenues pour une inclusion. Les inclusions représentées sont issues d'un premier tri destiné à éliminer les pollutions et les effets de pépite (Dillmann et L'Héritier, 2007; Disser et al., 2014). Les points identifiés comme " sélection " correspondent aux SI sélectionnées pour l'analyse des éléments traces et terres rares par LA-ICP-MS.

Figure 8: (See colour plate VIII) $\mathrm{Al}_{2} \mathrm{O}_{3} / \mathrm{SiO}_{2}$ and $\mathrm{K} 2 \mathrm{O} / \mathrm{CaO}$ ratios of the 4 analyzed semi-products (expressed in weight \%). Every dot represents the content obtained for one inclusion. The represented inclusions arise from a first sorting designed to eliminate pollutions and local concentration effects. (Dillmann et L'Héritier, 2007; Disser et al., 2014). Dots identified as "selection" correspond to the SI selected for trace element and rare earth analysis by LA-ICP-MS.

s'affranchir de potentiels effets d'enrichissement, les teneurs moyennes mesurées ont été normalisées à la teneur en $\mathrm{FeO}$ selon la formule proposée par Pagès (Pagès et al., 2011). Ces teneurs pondérées en fonction de la surface des inclusions et normalisées à la teneur en $\mathrm{FeO}$ seront par la suite marquées d'une double * (\%**).

On observe alors que DUR.40 se caractérise par une faible teneur en éléments autres qu' $\mathrm{Al}_{2} \mathrm{O}_{3}, \mathrm{SiO}_{2}, \mathrm{~K}_{2} \mathrm{O}$ et 
$\mathrm{CaO}(<0,5 \%$ mass $)$ et notamment par l'absence de $\mathrm{MnO}$ (tableau 2). En revanche, les inclusions sont riches en phosphore et cela vient confirmer les constatations faites en microscopie optique quant à la présence de structures "fantômes » de phosphore dans la ferrite. Elles présentent une teneur moyenne en $\mathrm{P}_{2} \mathrm{O}_{5}$ de $4,2 \% *$ et de $14,2 \%$ **.

Pour DUR.48, qui est également composé de deux masses métalliques soudées entre elles, les inclusions analysées sur les deux masses présentent des rapports NRC nettement distincts (figure 8 ). Ces signatures très différentes attestent que ce demi-produit a été élaboré à partir de deux masses de métal issues de deux ateliers de réduction différents et possiblement à partir de deux minerais distincts. Pour DUR.48-1, le taux de $\mathrm{MnO}$ est significativement élevé $(1,3 \% *$ et $3,4 \% * *)$ tandis que le taux de $\mathrm{P}_{2} \mathrm{O}_{5}$ reste faible $\left(0,5 \%^{*}\right.$ et $\left.1,2 \% * *\right)$. DUR.48-2 possède à la fois un taux de $\mathrm{MgO}$ élevé $\left(3,5 \%^{*}\right.$ et 7,42\%**) et un taux de $\mathrm{MnO}$ tout aussi significativement élevé $(1,4 \% *$ et $\left.3 \%{ }^{* *}\right)$. Les teneurs en $\mathrm{P}_{2} \mathrm{O}_{5}$ des inclusions corroborent la présence des structures fantômes observées au microscope optique $\left(2,6 \%^{*}\right.$ et $\left.6,5 \% * *\right)$.

Pour les deux autres demi-produits, aucune soudure n'est visible en métallographie et pour chaque exemplaire une unique signature chimique en NRC est décelable (figure 8). DUR.3 et DUR.6 se caractérisent tous deux par une forte teneur en $\mathrm{MnO}$, respectivement de 1,2\%* et 3,6\%** et de $2,4 \% *$ et $6,3 \%{ }^{* *}$. En revanche, seul DUR.3 possède une teneur significativement élevée en $\mathrm{P}_{2} \mathrm{O}_{5}\left(3,8 \% *\right.$ et $\left.10 \% \%^{* *}\right)$ ce qui confirme également leur différence de structure métallographique.

Au sein de chacune des parties de demi-produits individualisées par une différence chimique en éléments majeurs, un nombre représentatif d'inclusions a été sélectionné afin d'être analysé par LA-ICP-MS (inclusions représentées en rouge et en vert sur les graphiques des résultats EDS - figure 8). Le nombre d'inclusions analysées peut varier en raison de contraintes techniques, notamment la taille du laser et la sensibilité de l'analyseur. Ainsi, dans le cas de DUR.40 et de la partie 1 de DUR.48, pour lesquels les inclusions sont de grande taille, 9 inclusions ont pu être analysées pour l'un et 10 pour l'autre. En revanche, les trois autres demi-produits (DUR.3 - DUR.6) ou parties de demiproduit (DUR.48-2) comportaient des inclusions majoritairement inférieures à $30 \mu \mathrm{m}$ et seules 3,4 et 2 inclusions ont pu être respectivement analysées. Les résultats d'analyse par LA-ICP-MS sont présentés dans le tableau 4.

\begin{tabular}{|c|c|c|c|c|c|c|c|c|c|c|c|c|c|c|c|c|c|c|}
\hline \multirow{3}{*}{ Masse métallique } & \multicolumn{3}{|c|}{ mass $\%$} & \multicolumn{3}{|c|}{ mass $\%$} & \multicolumn{3}{|c|}{ mass $\%$} & \multicolumn{3}{|c|}{ mass $\%$} & \multicolumn{3}{|c|}{ mass $\%$} & \multicolumn{3}{|c|}{ mass $\%$} \\
\hline & \multicolumn{3}{|c|}{$\mathrm{NaO}$} & \multicolumn{3}{|c|}{$\mathrm{MgO}$} & \multicolumn{3}{|c|}{$\mathrm{Al}_{2} \mathrm{O}_{3}$} & \multicolumn{3}{|c|}{$\mathrm{SiO}_{2}$} & \multicolumn{3}{|c|}{$\mathrm{P}_{2} \mathrm{O}_{5}$} & \multicolumn{3}{|c|}{$\mathrm{SO}_{2}$} \\
\hline & $\%$ & \%* & $\%^{* *}$ & $\%$ & \%* & $\%^{* *}$ & $\%$ & \%* & $\%^{* *}$ & $\%$ & $\% *$ & $\%^{* *}$ & $\%$ & \%* & $\%^{* *}$ & $\%$ & \%* & $\%^{* *}$ \\
\hline DUR.40 & 0,2 & 0,2 & 0,6 & 0,3 & 0,3 & 0,8 & 3,6 & 4,1 & 12,1 & 16,2 & 17,9 & 52,5 & 4,3 & 4,8 & 14,2 & 0,3 & 0,2 & 0,7 \\
\hline DUR.48-1 & 0,1 & 0,1 & 0,1 & 0,5 & 0,6 & 1,5 & 4,5 & 5,1 & 11,8 & 28,3 & 31,3 & 72,0 & 0,4 & 0,5 & 1,2 & 0,2 & 0,1 & 0,3 \\
\hline DUR.48-2 & 0,3 & 0,4 & 0,6 & 3,5 & 4,2 & 7,4 & 2,2 & 3,0 & 5,4 & 27,4 & 32,5 & 57,2 & 2,6 & 3,7 & 6,5 & 0,2 & 0,2 & 0,4 \\
\hline DUR.3 & 0,2 & 0,2 & 0,5 & 0,3 & 0,3 & 0,8 & 2,2 & 2,4 & 6,0 & 24,9 & 24,5 & 60,9 & 3,8 & 4,0 & 10,0 & 0,2 & 0,2 & 0,4 \\
\hline DUR.6 & 0,1 & 0,1 & 0,3 & 0,4 & 0,4 & 1,1 & 7,4 & 7,4 & 18,9 & 25,0 & 25,3 & 64,5 & 0,5 & 0,4 & 1,1 & 0,4 & 0,4 & 0,9 \\
\hline \multirow{3}{*}{ Masse métallique } & \multicolumn{3}{|c|}{ mass $\%$} & \multicolumn{3}{|c|}{ mass $\%$} & \multicolumn{3}{|c|}{ mass $\%$} & \multicolumn{3}{|c|}{ mass $\%$} & \multicolumn{3}{|c|}{ mass $\%$} & \multicolumn{3}{|c|}{ mass $\%$} \\
\hline & \multicolumn{3}{|c|}{$\mathrm{K}_{2} \mathrm{O}$} & \multicolumn{3}{|c|}{$\mathrm{CaO}$} & \multicolumn{3}{|c|}{$\mathrm{TiO}$} & \multicolumn{3}{|c|}{$\mathrm{V}_{2} \mathrm{O}_{3}$} & \multicolumn{3}{|c|}{$\mathrm{MnO}$} & \multicolumn{3}{|c|}{$\mathrm{FeO}$} \\
\hline & $\%$ & $\%^{*}$ & $\% \%^{* *}$ & $\%$ & \%* & $\% * *$ & $\%$ & \%* & $\%^{* *}$ & $\%$ & $\% *$ & $\%^{* *}$ & $\%$ & $\% *$ & $\% * *$ & $\%$ & $\%^{*}$ & $\%^{* *}$ \\
\hline DUR.40 & 1,9 & 2,1 & 6,2 & 3,3 & 3,7 & 10,8 & 0,4 & 0,3 & 0,9 & 0,3 & 0,1 & 0,4 & 0,4 & 0,3 & 0,8 & 68,8 & 66,0 & 0,0 \\
\hline DUR.48-1 & 1,4 & 1,6 & 3,8 & 2,1 & 2,3 & 5,4 & 0,2 & 0,1 & 0,3 & 0,2 & 0,1 & 0,2 & 1,3 & 1,5 & 3,4 & 60,8 & 56,5 & 0,0 \\
\hline DUR.48-2 & 1,1 & 1,3 & 2,3 & 8,0 & 9,3 & 16,4 & 0,2 & 0,2 & 0,3 & 0,3 & 0,3 & 0,5 & 1,4 & 1,7 & 2,9 & 52,6 & 43,2 & 0,0 \\
\hline DUR.3 & 1,8 & 1,8 & 4,5 & 5,0 & 5,3 & 13,1 & 0,2 & 0,1 & 0,2 & 0,2 & 0,0 & 0,1 & 1,2 & 1,5 & 3,6 & 59,9 & 59,7 & 0,0 \\
\hline DUR.6 & 1,7 & 1,7 & 4,3 & 0,7 & 0,6 & 1,6 & 0,3 & 0,3 & 0,7 & 0,1 & 0,1 & 0,2 & 2,4 & 2,5 & 6,3 & 61,0 & 60,7 & 0,0 \\
\hline
\end{tabular}

$\%^{*}$ : moyenne arithmétique pondérée à la surface sur l'ensemble des inclusions

$\%^{* *}$ : moyenne pondérée à la surface et normalisée à $\mathrm{FeO}$

\%*: surface weighted average composition over all inclusions

$\%^{* *}$ : surface weighted average composition normalized to $\mathrm{FeO}$

Tableau 2 : Valeurs moyennes des éléments majeurs obtenues par MEB-EDS et pris en compte $\left(\mathrm{NRC}+\mathrm{MnO}_{\text {et }} \mathrm{P}_{2} \mathrm{O}_{5}\right)$ pour les 5 différentes masses de métal analysées. En gris, présence significative de l'élément.

Table 2: Average content of the major elements obtained by MEB-EDS and taken into account $\left(\mathrm{NRC}+\mathrm{MnO}\right.$ and $\left.\mathrm{P}_{2} \mathrm{O}_{5}\right)$ for the 5 analyzed different metal masses. Grey color indicates significant presence of the element. 
Dans un premier temps, une ACP a été menée sur les teneurs en éléments traces des inclusions après normalisation en rapport de logarithmes selon les paramètres décrits dans le chapitre 3.2. Sur la représentation des trois premiers axes de projection de l'ACP, 92,7 \% de la variance sont représentés (figure 9). Quatre groupes d'inclusions semblent se distinguer sur la figure. Le premier est formé des 2 masses DUR. 3 et DUR. 40 et les 3 autres par les 3 individus DUR.6 et DUR.48-1 et DUR.48-2. Les différences internes à chaque masse métallique individualisée (différences entre les signatures chimiques de chacune des inclusions d'une même masse) semblent plus faibles que les différences existantes entre les 5 masses métalliques analysées. Toutefois, sur ces représentations, les signatures des masses DUR.3 et DUR.40 semblent proches.

En revanche, la $\mathrm{CAH}$, réalisée sur les données $X_{E}$ tel que présenté dans le chapitre 3.2, permet une classification des inclusions et montre une dissimilarité plus grande entre DUR.40 et DUR.3 qu'entre les deux masses métalliques composant DUR.48 (DUR.48-1 et 48-2 - figure 10). Il est ainsi impossible de relier ces deux masses à une origine commune, d'autant plus si l'on prend en compte la différence observée plus haut au niveau des éléments majeurs et notamment la présence d'un taux de $\mathrm{MnO}$ significativement élevé dans les inclusions de DUR.3 et sa quasi-absence des inclusions de DUR.40.

Il semblerait donc que les cinq masses métalliques identifiées dans les quatre demi-produits analysés du dépôt de Durrenentzen soient issus de cinq origines différentes.
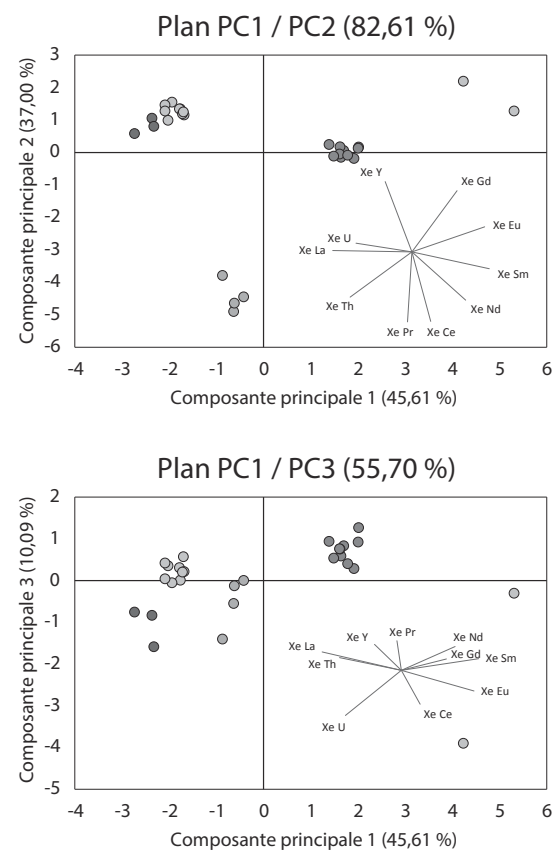

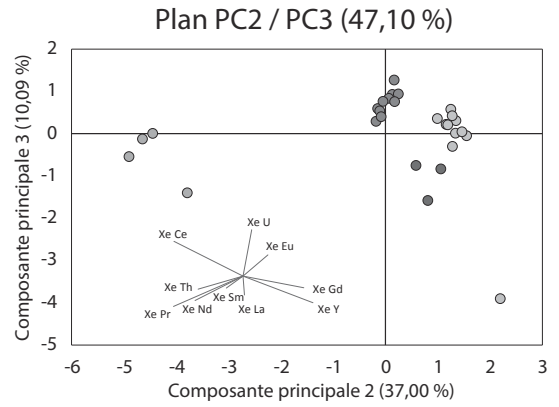

-DUR. 3 ○DUR. 40 ๑DUR. 48-1 ○ DUR. 48-2 ○DUR. 6

\section{Résultats des datations par le radiocarbone}

Comme cela a été précisé en introduction, le recours à une datation absolue du métal pour proposer une chronologie des bipyramidés apparaît actuellement indispensable, en raison de la pauvreté des informations fournies par les contextes archéologiques qui ont conduit à leur attribution au premier âge du Fer ou à l'époque gallo-romaine.

En suivant l'approche décrite précédemment, il a été possible de réaliser des datations pour chaque demi-produit après avoir prélevé dans les zones des coupes transversales qui contiennent localement suffisamment de carbone $\left(>0,02 \%_{\text {mass }} \mathrm{C}\right)$. Pour les objets constitués par l'assemblage de deux masses de métal, on a pris soin de ne prélever que dans une seule des deux masses.

Le tableau 3 présente les résultats des datations réalisées pour chacun des demi-produits. L'ensemble des distributions d'âges et le résultat des combinaisons des deux mesures obtenues à l'échelle de chaque masse métallique datée sont représentés dans la figure 11. La plupart des âges calibrés sont cohérents dans l'intervalle déterminé par l'incertitude à deux sigmas, et se regroupent autour du plateau du Premier âge du fer. On note une grande cohérence des datations effectuées au sein d'un même objet, ce qui permet de consolider leur probabilité. Seule la datation de DUR.3 livre deux distributions d'âge qui ne se recoupent pas dans l'intervalle à deux sigmas, l'une située autour de 900-800 calBC, l'autre située sur le plateau du Hallstatt (756-412 calBC). Pour les deux échantillons de DUR.3, la quantité de carbone extraite est suffisamment importante $(>1 \mathrm{mg})$ et l'analyse des $\delta^{13} \mathrm{C}$ est ici cohérente pour attester de la fiabilité des résultats. Le décalage de la mesure radiocarbone de DUR3-2 vers des dates plus anciennes pourrait être compatible avec un effet " vieux bois ». Une autre explication possible est que la présence d'un plateau sur la courbe de calibration autour de
Figure 9: (Voir planche couleur IX) Plans de plus grande variance (composantes principales 1,2 et 3) de l'Analyse en Composantes Principales réalisée sur les $X_{E}$ des 4 demi-produits en fer analysés.

Figure 9: (See colour plate IX) Planes of highest variance (principal components 1, 2 and 3) of the Principal Component Analysis realized on the $X_{E}$ of the 4 analyzed iron semi-products. 


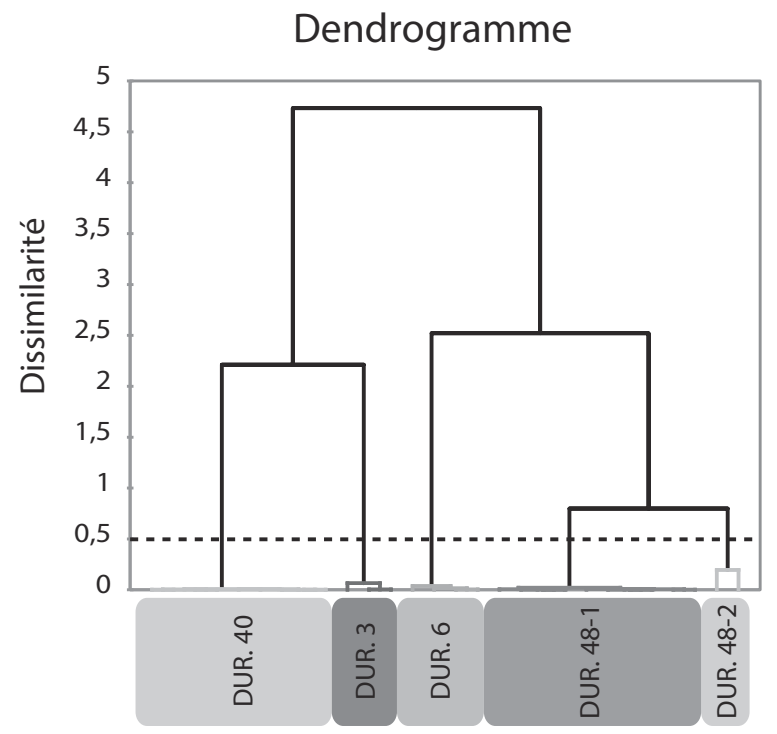

Figure 10 : (Voir planche couleur IX) Dendrogramme représentant les résultats de la Classification Ascendante Hiérarchique réalisée sur les $X_{\mathrm{E}}$ des 4 demi-produits en fer analysés.

Figure 10: (See colour plate IX) Dendrogram representing the results of the Hierarchical Agglomerative Clustering realized on the $X_{E}$ of the 4 analyzed iron semi-products.

2700 BP aurait tendance à étendre la densité d'âge vers des dates plus anciennes, et ne permettrait donc pas un étalonnage précis.

Néanmoins, les datations permettent toutes une attribution de ces objets au premier âge du fer, au Hallstatt $\mathrm{D} / \mathrm{La}$ Tène A. Dans la mesure où la courbe de calibration pour la période considérée présente un plateau entre -800 et - 420 il est impossible d'établir une datation plus précise. Les résultats de ces datations absolues permettent cependant d'ex- clure une attribution romaine pour ce dépôt. DUR.40, daté autour de -800 (835-792 calBC), pourrait être légèrement plus ancien que les autres.

\section{DisCussion}

Le lot de cinquante et un demi-produits mis au jour à Durrenentzen présente une grande homogénéité morphologique et métrologique, qui a conduit à deux interrogations principales. Cette homogénéité se retrouve-t-elle d'un point de vue interne (propriétés microscopiques et chimiques)? Quelles informations les propriétés des demi-produits permettent-elles d'obtenir sur la circulation du métal?

L'analyse métallographique met en évidence des différences significatives entre les quatre demi-produits étudiés à l'échelle interne, bien que la qualité d'épuration et la nature de l'alliage soient homogènes au sein d'un même demi-produit, même constitué de deux masses de métal différentes. Ces disparités concernent d'une part la qualité d'épuration qui s'avère fortement variable d'un objet à l'autre; d'autre part, elles concernent les techniques de fabrication, avec deux exemplaires résultant de l'assemblage de masses de métal et deux autres obtenus par déformation sur elle-même d'une seule masse métallique; et enfin, elles concernent la composition chimique du métal, avec trois familles d'alliages distincts caractérisés. Deux demi-produits sont ainsi constitués de fer phosphoreux, un autre est majoritairement ferritique et un dernier est majoritairement aciéré. On notera que ces différences de structures confèrent un comportement mécanique et des qualités de ductilité et de résistance propres à chacun des demi-produits. Cette hétérogénéité entre les demi-produits se retrouve également dans les signa-

\begin{tabular}{|c|c|c|c|c|c|c|}
\hline \multirow{2}{*}{ Prélèvement } & Code Lab. & \multirow{2}{*}{$\begin{array}{l}\text { Masse de carbone } \\
\text { extraite (mg) }\end{array}$} & \multirow{2}{*}{$d 13 \mathrm{C}(\% 0)$} & \multirow{2}{*}{$\mathrm{pMC}$} & \multirow{2}{*}{$\begin{array}{c}\text { Âge radiocarbone BP } \\
+/-(1 \sigma)\end{array}$} & \multirow{2}{*}{$\begin{array}{c}\text { Âge calibré } \\
(95,4 \%)\end{array}$} \\
\hline & (SacA) & & & & & \\
\hline DUR.40(1) & 26691 & 1,57 & $-30,4$ & $71,35 \pm 0,32$ & $2712 \pm 36$ & $923-804 \mathrm{BC}$ \\
\hline DUR.40(2) & 26692 & 1,5 & $-27,6$ & $72,46 \pm 0,28$ & $2588 \pm 31$ & $821-594 \mathrm{BC}$ \\
\hline DUR.48(1) & 26693 & 1,2 & $-23,9$ & $73,26 \pm 0,28$ & $2499 \pm 31$ & $786-515 \mathrm{BC}$ \\
\hline DUR.48(2) & 26694 & 0,87 & $-28,9$ & $73,58 \pm 0,30$ & $2465 \pm 33$ & $761-414 \mathrm{BC}$ \\
\hline DUR.3(1) & 26695 & 1,45 & $-18,3$ & $73,65 \pm 0,29$ & $2457 \pm 32$ & $756-412 \mathrm{BC}$ \\
\hline DUR.3(2) & 26696 & 1,42 & $-22,8$ & $71,71 \pm 0,33$ & $2671 \pm 37$ & $900-796 \mathrm{BC}$ \\
\hline DUR.6(1) & 26697 & 0,3 & $-37,6$ & $73,14 \pm 0,33$ & $2513 \pm 36$ & $792-521 \mathrm{BC}$ \\
\hline DUR.6(2) & 26698 & 0,86 & $-29,9$ & $73,86 \pm 0,30$ & $2434 \pm 33$ & $752-404 \mathrm{BC}$ \\
\hline
\end{tabular}

Tableau 3 : Résultats des datations réalisées sur les demi-produits en fer du dépôt de Durrenentzen. Calibration effectuée grâce au logiciel Oxcal 4.2.4 (Ramsey, 2013) qui utilise la courbe de calibration IntCal 13 (Reimer et al., 2013).

Table 3: Results of the $14 \mathrm{C}$ dating obtained on the iron semi-products of the Durrenentzen deposit. Calibration made using the software Oxcal 4.2.4 (Ramsey, 2013) which uses the calibration curve IntCal 13 (Reimer et al., 2013). 
Figure 11 : (Voir planche couleur V) Distributions d'âge (à 95,4 \% de probabilité) des deux mesures $14 \mathrm{C}$ obtenues pour chacun des 4 demi-produits en fer et combinaisons (en gris) des deux mesures associées à l'âge du bois utilisé pour leurs fabrications.

Figure 11: (See colour plate V) Age Distributions (with 95.4\% of probability) of the two $14 \mathrm{C}$ measures obtained for each of the 4 iron semi-products and combination (in grey) of the two measures associated with the age of the wood used for their manufacturing.

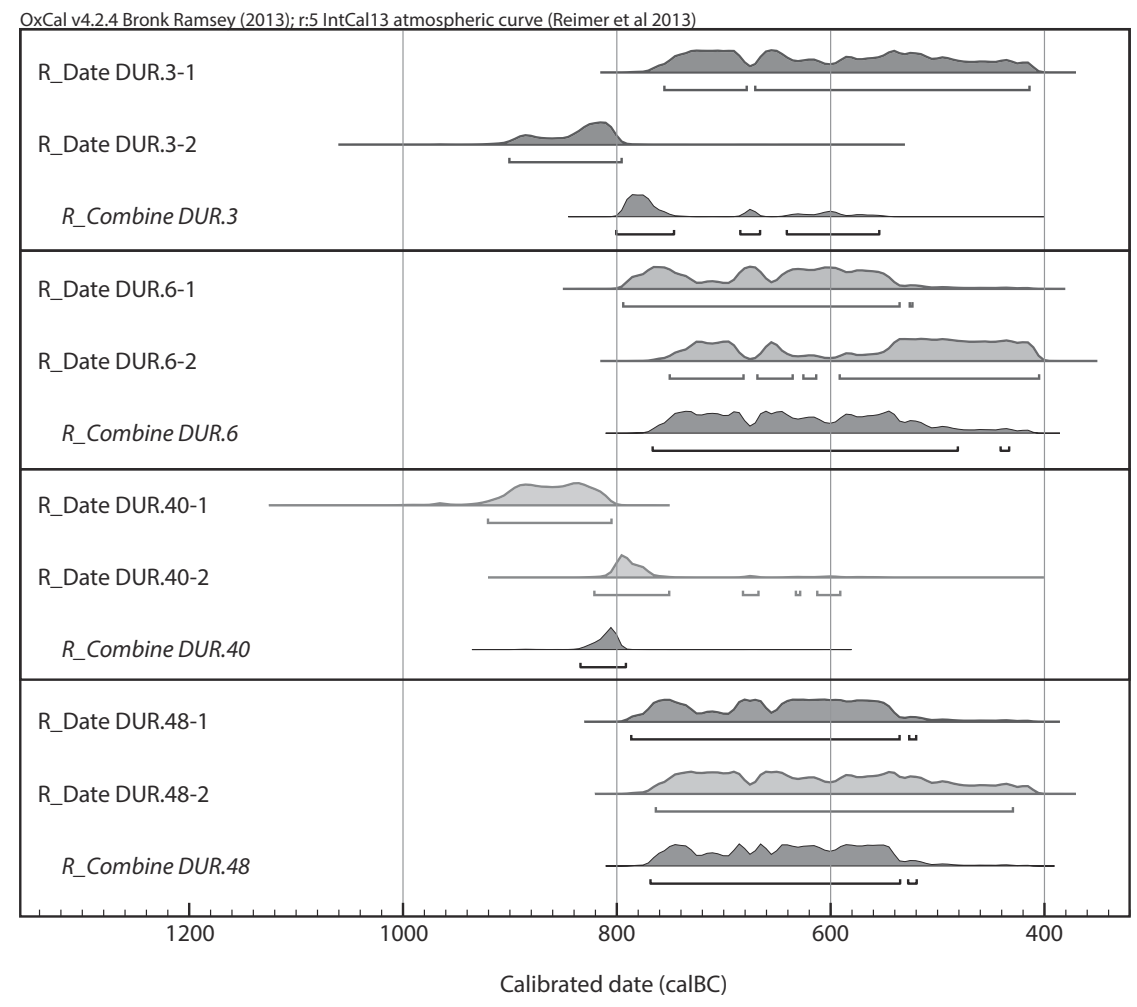

tures chimiques. L'analyse chimique inclusionnaire réalisée sur six masses de métal distinctes révèle l'existence de cinq signatures chimiques différenciées.

Cette pluralité des signatures chimiques au sein d'un même objet est également connue par l'analyse inclusionnaire de demi-produits plus tardifs, les barres à douille datées de la fin de l'âge du Fer (Bauvais et Fluzin, 2006; Berranger et al., 2007). Elle a permis de révéler un système d'échange complexe, caractérisé par une segmentation de la chaîne opératoire (séparation des activités de réduction, de fabrication de demi-produits et de fabrication d'objets), également documentée au sein des ateliers (Bauvais, 2008; Berranger, 2014). Les présents résultats permettent d'attribuer à des périodes bien plus anciennes cette segmentation de la chaîne-opératoire avec une spécialisation entre artisans, alors qu'elle est peu perceptible par la seule étude des ateliers contemporains.

Effectivement, les datations radiocarbones permettent de dater cet ensemble durant une période précoce du développement des activités sidérurgiques, celle du Hallstatt-La Tène A. Elles confirment celles déjà obtenues sur des microcharbons de bois conservés dans les porosités de deux bipyramidés issus d'un dépôt de vingt-quatre demi-produits (Nottonville, Eure et Loire, Berranger, 2014, p. 115-116 et 147). Les sites livrant des bipyramidés datés par les contextes archéologiques, sont jusqu'à présent majoritairement associés au Hallstatt D-La Tène A (Berranger, 2014, fig. 35). Ces résultats apportent ainsi des arguments supplémentaires en faveur d'une datation précoce des bipyramidés. L'étude interdisciplinaire menée sur les demi-produits de Durrenentzen permet d'ores et déjà d'attribuer leurs caractéristiques techniques au contexte chrono-culturel de la fin du premier âge du Fer.

Le dépôt, qui rassemble plus de $308 \mathrm{~kg}$ de fer épuré, révèle la circulation de quantités considérables de métal, alors que pour cette période les objets finis ne sont connus qu'en faibles proportions dans les sites de consommation. Parmi une quinzaine de sites du Hallstatt D dont le mobilier métallique a été récemment étudié, le vaste complexe d'habitat de la Heuneburg (Allemagne) est le seul à se démarquer par des quantités conséquentes d'objets en fer, ne dépassant néanmoins pas $12 \mathrm{~kg}$ (Dubreucq, 2013, fig. 103). Les autres habitats ne livrent généralement pas plus de $1 \mathrm{~kg}$ de produits ferreux.

La présente étude révèle l'assemblage de masses de métal d'origines distinctes au sein d'un unique demi-produit. Ceci met en évidence la circulation de la matière première sous une forme brute, non, voire peu épurée, directement depuis les ateliers de réduction. Ce constat, déjà mis en avant par Gaspard Pagès pour l'époque gallo-romaine, sur les barres 


\begin{tabular}{|c|c|c|c|c|c|c|c|c|c|c|c|c|c|}
\hline \multicolumn{2}{|c|}{ Masse métallique } & DUR.3 & DUR.3 & DUR.3 & DUR.40-1 & DUR.40-1 & DUR.40-1 & DUR.40-2 & DUR.40-2 & DUR.40-2 & DUR.40-2 & DUR.40-2 & DUR.40-2 \\
\hline \multicolumn{2}{|c|}{ Inclusion } & 1 & 2 & 3 & 1 & 2 & 3 & 4 & 5 & 6 & 7 & 8 & 9 \\
\hline \multirow{37}{*}{ 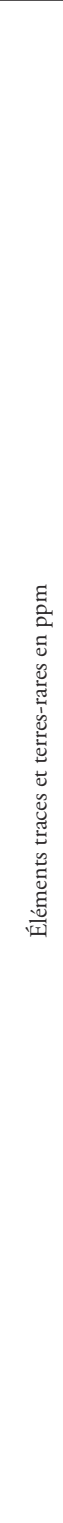 } & As & 40,3 & 685,7 & 2,9 & 5,2 & 14,7 & 8,3 & 17,1 & 3,6 & nd & 1,4 & 2,9 & 9,2 \\
\hline & $\mathrm{Ba}$ & 13067,0 & 11155,2 & 13037,9 & 1763,4 & 2025,1 & 2105,9 & 2425,4 & 2254,0 & 2539,4 & 2345,4 & 2053,8 & 2440,8 \\
\hline & $\mathrm{Be}$ & 161,2 & 114,2 & 127,7 & 275,1 & 299,1 & 307,1 & 377,2 & 358,3 & 377,1 & 359,2 & 328,8 & 376,7 \\
\hline & $\mathrm{Ce}$ & 89,5 & 74,9 & 86,3 & 190,9 & 211,5 & 230,4 & 211,4 & 241,4 & 276,0 & 242,5 & 219,7 & 269,1 \\
\hline & Co & 49,4 & 1366,9 & 2,5 & 7,6 & 18,7 & 6,1 & 24,7 & 32,0 & 22,9 & 16,0 & 32,4 & 74,7 \\
\hline & $\mathrm{Cr}$ & nd & nd & nd & 448,9 & 817,8 & 497,1 & 609,1 & 551,8 & 537,6 & 340,9 & 658,4 & 871,6 \\
\hline & $\mathrm{Cs}$ & 0,2 & nd & 4,0 & 5,1 & 4,3 & 6,0 & 8,8 & 0,9 & 1,5 & 2,2 & 1,5 & 2,9 \\
\hline & $\mathrm{Cu}$ & 40,4 & 1081,3 & 4,6 & 39,6 & 22,0 & 27,5 & 101,8 & 44,5 & 14,7 & 19,7 & 83,3 & 133,1 \\
\hline & $\mathrm{Eu}$ & 7,9 & 5,6 & 6,5 & 18,0 & 19,1 & 22,6 & 20,8 & 21,3 & 26,2 & 23,5 & 17,6 & 24,7 \\
\hline & $\mathrm{Ga}$ & 2033,5 & 1377,5 & 2070,6 & 335,0 & 382,1 & 408,7 & 450,7 & 414,6 & 460,4 & 428,2 & 369,5 & 442,9 \\
\hline & $\mathrm{Gd}$ & 40,2 & 24,2 & 33,4 & 86,2 & 99,1 & 114,2 & 103,2 & 111,2 & 130,0 & 117,3 & 111,7 & 133,7 \\
\hline & $\mathrm{Ge}$ & 11,0 & 50,0 & 4,4 & 19,9 & 27,0 & 33,9 & 24,8 & 33,6 & 41,1 & 35,5 & 28,5 & 37,4 \\
\hline & $\mathrm{Hf}$ & 3,8 & 2,7 & 5,5 & 8,3 & 10,7 & 11,2 & 9,7 & 10,4 & 11,9 & 10,0 & 10,3 & 11,2 \\
\hline & $\mathrm{La}$ & 192,4 & 126,3 & 173,0 & 434,5 & 455,0 & 551,8 & 520,4 & 569,3 & 658,0 & 577,1 & 512,1 & 697,1 \\
\hline & Mo & 2,8 & 16,0 & 0,9 & 0,3 & 0,4 & 0,8 & 1,4 & 0,9 & 4,1 & 1,5 & nd & nd \\
\hline & $\mathrm{Nb}$ & 5,6 & 5,4 & 8,6 & 23,0 & 24,8 & 24,8 & 26,2 & 25,1 & 29,1 & 26,5 & 23,1 & 30,7 \\
\hline & $\mathrm{Nd}$ & 127,2 & 86,3 & 115,3 & 275,8 & 271,3 & 340,9 & 299,7 & 361,3 & 403,0 & 360,0 & 334,2 & 421,7 \\
\hline & $\mathrm{Ni}$ & 363,4 & 5500,9 & 8,3 & 7,3 & 3,7 & 6,5 & 24,4 & 15,9 & 4,8 & 11,9 & 11,7 & 51,9 \\
\hline & $\mathrm{Pb}$ & 1,4 & 21,1 & 0,4 & 8,0 & 11,6 & 9,7 & 17,5 & 7,5 & 10,1 & 4,8 & 8,6 & 28,5 \\
\hline & $\mathrm{Pd}$ & 0,1 & 0,2 & nd & 1,2 & 0,7 & 0,1 & nd & nd & 0,7 & 0,7 & 0,9 & 0,4 \\
\hline & $\operatorname{Pr}$ & 27,8 & 20,2 & 24,7 & 55,1 & 59,8 & 74,4 & 64,4 & 73,7 & 87,8 & 77,8 & 66,5 & 85,2 \\
\hline & $\mathrm{Rb}$ & 42,4 & 64,9 & 96,5 & 88,3 & 96,6 & 113,8 & 164,9 & 117,6 & 132,0 & 106,0 & 108,3 & 137,2 \\
\hline & $\mathrm{Sb}$ & 0,8 & 10,3 & nd & nd & 0,9 & 1,1 & 3,0 & nd & nd & nd & 0,8 & 0,3 \\
\hline & $\mathrm{Sc}$ & 27,3 & 18,4 & 23,0 & 39,8 & 49,1 & 46,9 & 51,5 & 50,0 & 46,4 & 40,4 & 48,6 & 56,4 \\
\hline & $\mathrm{Sm}$ & 26,4 & 15,0 & 22,9 & 63,7 & 68,8 & 75,8 & 71,2 & 79,9 & 106,9 & 86,4 & 76,3 & 88,1 \\
\hline & Sn & 7,4 & 16,9 & 13,7 & 41,4 & 52,6 & 48,7 & 50,7 & 38,3 & 39,5 & 34,6 & 35,7 & 92,8 \\
\hline & $\mathrm{Sr}$ & 287,4 & 316,2 & 320,8 & 668,7 & 714,2 & 808,5 & 827,1 & 872,4 & 963,7 & 855,6 & 789,2 & 918,5 \\
\hline & $\mathrm{Ta}$ & 0,7 & 1,0 & 0,7 & 1,5 & 1,7 & 1,6 & 1,8 & 2,0 & 1,8 & 1,8 & 1,9 & 1,9 \\
\hline & $\mathrm{Tb}$ & 5,6 & 3,6 & 5,1 & 15,9 & 17,7 & 20,1 & 17,9 & 21,1 & 25,9 & 22,5 & 18,0 & 26,3 \\
\hline & Th & 11,9 & 5,2 & 12,4 & 26,1 & 30,8 & 33,3 & 34,1 & 39,0 & 40,3 & 35,5 & 30,0 & 41,8 \\
\hline & $\mathrm{U}$ & 169,9 & 173,1 & 149,6 & 46,2 & 50,0 & 50,4 & 57,9 & 61,8 & 67,2 & 64,3 & 52,6 & 69,0 \\
\hline & $\mathrm{V}$ & 27,8 & 36,1 & 223,6 & 137,4 & 190,5 & 137,9 & 173,5 & 197,4 & 184,3 & 146,1 & 194,0 & 261,7 \\
\hline & W & 0,2 & 1,9 & 0,1 & 5,3 & 6,6 & 6,6 & 4,2 & 5,8 & 4,7 & 4,7 & 3,1 & 4,0 \\
\hline & $\mathrm{Y}$ & 478,9 & 293,1 & 414,4 & 1145,4 & 1282,2 & 1463,8 & 1356,3 & 1451,0 & 1658,6 & 1452,3 & 1341,9 & 1792,9 \\
\hline & $\mathrm{Yb}$ & 22,7 & 14,5 & 19,3 & 69,1 & 75,0 & 80,2 & 79,0 & 92,5 & 107,9 & 86,3 & 84,1 & 102,2 \\
\hline & $\mathrm{Zn}$ & 42,5 & 658,8 & 22,0 & 42,7 & 59,4 & 48,4 & 106,5 & 45,4 & 35,5 & 31,5 & 71,1 & 192,2 \\
\hline & $\mathrm{Zr}$ & 157,5 & 81,1 & 207,0 & 347,0 & 369,9 & 385,2 & 380,8 & 369,7 & 428,2 & 382,8 & 362,2 & 612,9 \\
\hline
\end{tabular}

Tableau 4 : Composition en éléments traces et terres rares des inclusions sélectionnées pour analyses chimiques globales par LA-ICP-MS. Les éléments pris en compte pour la définition de la signature chimique sont surlignés en gris.

Table 4: Composition in trace elements and rare earth elements of each slag inclusion selected for full chemical analysis by LA-ICP-MS. Grey color indicates the elements taken into account to define the chemical signature.

des Saintes-Maries-de-la-Mer (Pagès, 2010; Pagès et al., 2011), confirme qu’à des périodes même très anciennes, le métal circule sous sa forme brute et qu'il n'est pas forcément compacté sur son lieu de réduction. La masse brute retrouvée à Bourges et datée de La Tène A en est un excellent complément (Fournier et Milcent, 2007) en accord avec les observations faites dans le cadre de ce travail. Il s'agit toutefois d'une forme d'échange du fer très peu attestée, en raison de l'absence quasi-générale de masses brutes de réduction conservées pour cette période. La diversité des signatures chimiques constatée au sein du lot étudié indiquerait que les artisans acquéraient leur matière première brute auprès de réseaux d'échanges variés. La mise en forme finale de ces produits aurait alors pu être effectuée au sein d'un atelier ou d'un ensemble d'ateliers de forgeage se conformant à un standard métrologique. C'est ce que permettrait d'en déduire 


\begin{tabular}{|c|c|c|c|c|c|c|c|c|c|c|c|c|c|c|c|c|c|}
\hline \multicolumn{2}{|c|}{\begin{tabular}{|l|} 
Masse \\
métal- \\
lique
\end{tabular}} & $\begin{array}{c}\text { DUR. } \\
48-1\end{array}$ & $\begin{array}{c}\text { DUR. } \\
48-1\end{array}$ & $\begin{array}{c}\text { DUR. } \\
48-1\end{array}$ & $\begin{array}{c}\text { DUR. } \\
48-1\end{array}$ & $\begin{array}{c}\text { DUR. } \\
48-1\end{array}$ & $\begin{array}{c}\text { DUR. } \\
48-1\end{array}$ & $\begin{array}{c}\text { DUR. } \\
48-1\end{array}$ & $\begin{array}{l}\text { DUR. } \\
48-1\end{array}$ & $\begin{array}{l}\text { DUR. } \\
48-1\end{array}$ & $\begin{array}{c}\text { DUR. } \\
48-1\end{array}$ & $\begin{array}{c}\text { DUR. } \\
48-2\end{array}$ & $\begin{array}{l}\text { DUR. } \\
48-2\end{array}$ & DUR. 6 & DUR.6 & DUR. 6 & DUR. 6 \\
\hline & 1 & 2 & 3 & 4 & 5 & 6 & 7 & 8 & 9 & 10 & 1 & 2 & 1 & 2 & 3 & 4 \\
\hline \multirow{37}{*}{ 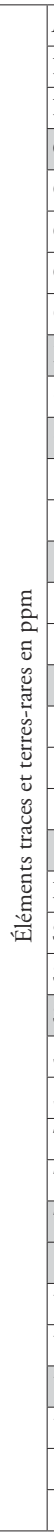 } & As & 6,8 & 26,4 & 14,2 & 31,7 & 12,0 & 49,9 & 1192,0 & 445,1 & 1401,3 & 44,2 & 70,6 & 31002,9 & 48,9 & 8,0 & 69,3 & 761,7 \\
\hline & $\mathrm{Ba}$ & 2368,7 & 1930,0 & 2292,1 & 2325,8 & 2595,1 & 3261,8 & 2726,2 & 2646,7 & 3850,5 & 3254,5 & 6723,2 & 2528,9 & 2266,7 & 2499,9 & 2144,3 & 1034,3 \\
\hline & $\mathrm{Be}$ & 123,8 & 112,9 & 131,0 & 120,2 & 123,6 & 141,1 & 132,7 & 146,8 & 94,6 & 143,7 & 195,8 & 117,4 & 29,1 & 43,0 & 28,4 & 28,5 \\
\hline & $\mathrm{Ce}$ & 263,0 & 238,1 & 300,0 & 276,2 & 250,7 & 317,2 & 330,6 & 303,4 & 321,5 & 328,3 & 378,2 & 201,6 & 713,9 & 638,9 & 630,8 & 320,6 \\
\hline & Co & 5,2 & 12,4 & 10,1 & 9,3 & 21,6 & 92,1 & 788,6 & 195,7 & 540,7 & 39,6 & 31,3 & 8750,0 & 43,9 & 1,2 & 26,7 & 4012,3 \\
\hline & $\mathrm{Cr}$ & nd & nd & nd & nd & nd & nd & nd & nd & nd & nd & nd & nd & 38,9 & 430,2 & 324,7 & 2022,0 \\
\hline & Cs & 17,5 & 12,3 & 13,3 & 7,7 & 9,1 & 15,7 & 21,5 & 8,9 & 14,3 & 2,3 & 26,6 & nd & 20,6 & 18,3 & 14,8 & 17,3 \\
\hline & $\mathrm{Cu}$ & 37,3 & 19,2 & 10,6 & 8,7 & 19,8 & 39,1 & 119,7 & 472,2 & 499,4 & 448,9 & 32,5 & 14938,8 & 504,8 & 4,5 & 321,2 & 38502,2 \\
\hline & Eu & 23,1 & 22,1 & 27,8 & 26,6 & 23,0 & 30,6 & 29,6 & 25,3 & 28,1 & 30,1 & 43,2 & 16,9 & 14,2 & 15,2 & 14,3 & 5,3 \\
\hline & Ga & 211,9 & 219,2 & 312,9 & 308,3 & 375,2 & 488,9 & 400,0 & 425,4 & 612,8 & 543,0 & 834,3 & 334,7 & 398,3 & 459,1 & 387,0 & 258,1 \\
\hline & $\mathrm{Gd}$ & 128,4 & 102,6 & 122,2 & 99,7 & 107,3 & 128,4 & 125,5 & 101,7 & 103,5 & 109,5 & 177,8 & 47,0 & 49,8 & 59,0 & 55,1 & 21,1 \\
\hline & $\mathrm{Ge}$ & 26,2 & 24,3 & 36,2 & 27,3 & 31,4 & 43,6 & 39,2 & 46,3 & 39,5 & 38,2 & 37,9 & 165,8 & 26,0 & 26,9 & 27,7 & 176,3 \\
\hline & Hf & 3,4 & & & & & & & & & 3,7 & & 17,5 & & 14 & 9 & 6,9 \\
\hline & $\mathrm{La}$ & 270,9 & 238,0 & 306,1 & 272,0 & 266,2 & 314,0 & 328,7 & 278,7 & 263,9 & 296,1 & 142,9 & 44,2 & 284,8 & 305,5 & 289,9 & 99,5 \\
\hline & Mo & 0,1 & 1,4 & nd & 0,2 & 0,4 & 0,5 & 4,8 & 2,4 & 5,1 & 2,1 & 0,8 & 74,5 & 2,0 & 1,9 & 2,7 & 234,1 \\
\hline & $\mathrm{Nb}$ & 14,3 & 12,8 & 15,6 & 13,9 & 15,1 & 19,2 & 17,5 & 14,0 & 13,7 & 14,8 & 7,0 & & 5,1 & 30,3 & 26,8 & 13,0 \\
\hline & $\mathrm{Nd}$ & 444,8 & 382,1 & 459,9 & 412,5 & 388,4 & 473,5 & 487,9 & 410,0 & 439,5 & 465,9 & 607,8 & 113,5 & 358,2 & 401,7 & 368,0 & 109,0 \\
\hline & $\mathrm{Ni}$ & 3,6 & 10,6 & 1,5 & 18,9 & 14,6 & 195,2 & 1011,2 & 622,3 & 2409,7 & 55,4 & 7,1 & 17404,6 & 118,3 & 1,7 & 89,4 & 3787,4 \\
\hline & $\mathrm{Pb}$ & 0,5 & 0,8 & 2,0 & 2,5 & 8,8 & 5,9 & 1,1 & 8,8 & 5,8 & 4,5 & 6,5 & 133,4 & 19,4 & 1,5 & 5,8 & 718,0 \\
\hline & $\mathrm{Pd}$ & 0,7 & 0,2 & 0,4 & 0,5 & 0,6 & 0,9 & 0,7 & 0,2 & 0,2 & 0,7 & 0,3 & nd & 0,3 & 0,5 & 0,6 & 1,1 \\
\hline & $\operatorname{Pr}$ & 78,1 & 67,9 & 84,5 & 76,4 & 71,6 & 87,5 & 91,6 & 77,3 & 79,1 & 84,4 & 76,8 & 14,4 & 85,3 & 87,5 & 83,7 & 26,8 \\
\hline & $\mathrm{Rb}$ & 152,3 & 90,0 & 125,6 & 100,2 & 100,1 & 136,9 & 145,3 & 116,0 & 134,7 & 121,2 & 138,9 & 25,4 & 311,0 & 233,5 & 197,5 & 113,6 \\
\hline & $\mathrm{Sb}$ & nd & nd & 0,3 & 0,5 & nd & nd & 5,0 & 3,1 & 7,3 & 1,2 & nd & 53,8 & 0,7 & 0,2 & 0,9 & 93,3 \\
\hline & Sc & 97,5 & 53,2 & 77,9 & 56,8 & 57,2 & 41,1 & 16,0 & nd & nd & nd & nd & 660,1 & 73,7 & 90,6 & 77,4 & 62,7 \\
\hline & Sm & 104,6 & 92,6 & 112,5 & 97,0 & 89,3 & 118,1 & 119,5 & 104,9 & 108,7 & 122,3 & 171,5 & 38,5 & 65,2 & 71,4 & 69,5 & 29,1 \\
\hline & Sn & 11,5 & 11,8 & 14,3 & 7,5 & 21,9 & 29,7 & 20,0 & 17,6 & 19,7 & 25,8 & 47,4 & 156,7 & 13,8 & 13,5 & 16,5 & 178,3 \\
\hline & $\mathrm{Sr}$ & 123,6 & 99,2 & 108,5 & 116,1 & 141,6 & 166,2 & 126,7 & 142,2 & 167,2 & 161,8 & 199,9 & 443,5 & 188,7 & 184,1 & 163,9 & 719,9 \\
\hline & $\mathrm{Ta}$ & 0,5 & 0,6 & 1,0 & 0,7 & 0,2 & 0,7 & 0,8 & 1,5 & 0,8 & 1,2 & nd & 19,5 & 1,3 & 1,7 & 1,9 & 66,5 \\
\hline & $\mathrm{Tb}$ & 15,2 & 13,0 & 16,7 & 14,0 & 14,4 & 17,4 & 17,3 & 15,1 & 15,8 & 18,2 & 23,0 & 5,8 & 7,3 & 8,8 & 8,0 & 2,9 \\
\hline & Th & 21,5 & 17,9 & 24,3 & 21,4 & 22,1 & 27,9 & 25,4 & 21,7 & 20,3 & 23,0 & 14,0 & 4,3 & 32,0 & 36,6 & 39,3 & 12,8 \\
\hline & $\mathrm{U}$ & 14,4 & 12,1 & 14,9 & 14,0 & 13,0 & 14,5 & 16,0 & 14,0 & 16,7 & 15,6 & 25,6 & 19,3 & 39,5 & 31,3 & 34,9 & 15,1 \\
\hline & $\mathrm{V}$ & 1077,5 & 706,8 & 740,7 & 771,5 & 643,0 & 1521,9 & 887,7 & 789,2 & 727,7 & 718,9 & 525,0 & 316,9 & 29,5 & 707,7 & 553,2 & 524,3 \\
\hline & W & nd & nd & nd & nd & 4,0 & 10,1 & 0,8 & 5,4 & 3,1 & 3,6 & nd & 149,6 & 0,6 & 0,1 & 0,9 & 151,0 \\
\hline & $\mathrm{Y}$ & 863,9 & 747,5 & 942,6 & 807,9 & 892,9 & 1048,5 & 1005,6 & 876,4 & 835,0 & 960,2 & 1039,2 & 185,3 & 238,5 & 293,9 & 264,7 & 109,2 \\
\hline & $\mathrm{Yb}$ & 63,6 & 55,0 & 64,2 & 55,3 & 57,0 & 74,9 & 70,3 & 55,3 & 54,4 & 57,6 & 69,7 & 9,6 & 23,0 & 27,4 & 25,4 & 10,1 \\
\hline & $\mathrm{Zn}$ & nd & 11,7 & 23,8 & 6,7 & 43,9 & 59,8 & 21,4 & 379,4 & 292,1 & 90,6 & nd & 7076,6 & 181,1 & 10,1 & 105,1 & 14393,1 \\
\hline & $\mathrm{Zr}$ & 507,5 & 448,5 & 569,4 & 469,3 & 540,3 & 678,7 & 625,3 & 740,4 & 461,4 & 576,6 & 356,1 & 3056,7 & 429,7 & 602,7 & 548,6 & 562,7 \\
\hline
\end{tabular}

Tableau 4 (suite)

Table 4 (following)

l'homogénéité morphologique globale du lot conjointement à l'existence de variations de détail.

La formation de certains demi-produits à partir de plusieurs masses de métal différenciées permet également d'envisager l'acquisition de portions de métal de volume variable, transformées ultérieurement de façon à se conformer à un standard métrologique. C'est également ce que pourraient indiquer les cassures des extrémités observées sur certains demi-produits. La forme de deux pyramides acco- lées ou de "saumon " est généralement considérée comme la morphologie résultant " naturellement » du resserrement sur elle-même d'une masse brute de réduction (Thouvenin, 1984). Des demi-produits d'autres espaces chrono-culturels connaissent d'ailleurs cette même morphologie du métal en circulation, comme les saumons Assyriens de Khorsabad, de Suse ou de Nimrud (Pleiner, 2006, p. 25) ou les demiproduits des épaves médiévales découvertes au large de Haïfa (Galili et al., 2015). Au sein de ces épaves, la présence de 
plusieurs stades de compaction des masses brutes illustre particulièrement bien le lien morpho-technologique entre cette forme de double pyramide et la compaction des masses brutes. La conformation à un standard des bipyramidés étudiés dans la présente étude, et le fait que certains soient obtenus à partir de masses de métal épurées individuellement et soudées entre-elles, montrent que cette forme ne correspond plus seulement à une résultante technique, mais aussi à un choix culturel et/ou économique, à une norme morphologique. Ces caractéristiques induisent ainsi l'existence d'un système de référence commun au stade de l'élaboration et de la diffusion de ces produits semi-manufacturés.

Pour les mêmes périodes, cette conformation à des standards communs est généralement bien connue pour la fabrication d'objets finis, telles que les fibules dont certaines connaissaient une production de masse (Dubreucq, 2013; Carrara et al., 2013). Elle est encore mieux cernée à partir de la céramique. Dans le domaine hallstattien, la période du Hallstatt D2/D3-LTA est ainsi celle qui voit la diffusion et l'adoption d'un nouveau système de fabrication des céramiques, celui du façonnage au tour. Pour les périodes les plus anciennes, ces céramiques tournées semblent réalisées au sein d'ateliers apparemment spécialisés, situés de façon récurrente à proximité des sites princiers (Augier et al., 2013). Ce même lien entre la répartition des bipyramidés et celles de ces centres de pouvoirs a déjà été notée (Berranger et Fluzin, 2012). Le dépôt de Durrenentzen se localise ainsi à seulement $10 \mathrm{~km}$ à vol d'oiseau de Breisach «Münsterhügel ", site princier du Hallstatt D/La Tène A (Balzer, 2009). Ces cas d'étude fournissent ainsi de nouveaux éléments d'analyse relatifs à l'importance des élites dans la diffusion des productions et des savoir-faire, mais permet aussi de mieux évaluer leur rôle moteur dans l'émergence de nouvelles spécialisations et structurations artisanales.

\section{Conclusions}

L'étude de ce dépôt exceptionnel de demi-produits permet d'implanter le premier jalon d'une analyse plus générale modifiant significativement la perception de l'économie du fer au premier âge de Fer.

Parfois négligée et à d'autres moments exagérée, la circulation du fer au cours des premiers temps de son utilisation dans le nord de l'Europe se voit ici pour la première fois documentée d'une façon nouvelle, depuis la circulation de masses brutes jusqu'à leur mise en forme et leur sortie des circuits économiques. En effet, les nouvelles approches archéométriques utilisées ont permis non seulement de rattacher ce dépôt à la période du Hallstatt (datation 14C du carbone dans les aciers) mais aussi de mettre en évidence le caractère composite de certains demi-produits et l'hétérogénéité des provenances du dépôt dans son ensemble. Il se dessine alors un modèle théorique d'une économie complexe, mettant en jeu de multiples acteurs à divers niveaux techniques et degrés d'implication (figure 12). La nature des relations entre ces acteurs semble également bien plus complexe qu'une simple relation directe entre producteur et consommateur, et la quantité de métal en circulation apparait beaucoup plus importante que ce que les sites d'habitat de cette période pouvaient laisser présager.

Ce premier jalon, de l'ordre du modèle théorique, ouvre à présent des perspectives nouvelles quant à l'interprétation de l'économie du fer aux âges du Fer. L'approche résolument interdisciplinaire développée au sein du LMC/LAPA-IRAMAT, qui a été appliquée à cet ensemble, doit à présent être étendue à d'autres dépôts du complexe Nord-Alpin afin de consolider les premières conclusions apportées par cette étude.

Enfin, une étape primordiale de l'approche reste à développer; l'étude de provenance à proprement parler (Leroy et al., 2012a; Leroy et al., 2014; Charlton, 2015; Disser et al., 2016). Une base de données géochimiques (éléments majeurs, traces et isotopes de l'Osmium) des espaces de production sidérurgiques du nord de la France et du sud de l'Allemagne est en cours de développement et réunit plusieurs travaux du LMC/LAPA-IRAMAT (ANR-DFG CIPIA, Thèse A. Disser 2014, PCR La sidérurgie en Bourgogne et en Franche-Comté avant le haut fourneau, Thèse N. Zaour en cours) (Bauvais et al., 2011; Bauvais et al., 2015; Berranger, 2015; Disser, 2014). La prochaine étape sera de confronter ces premiers résultats aux espaces de production potentiels ce qui permettra de relier enfin des centres de productions à des espaces de consommation et d'ajouter à ce modèle théorique en construction des relations géoéconomiques et géopolitiques cruciaux pour une compréhension globale de l'économie du fer protohistorique.

\section{Remerciements}

Nous tenons à remercier le musée de Biesheim et sa conservatrice Suzanne Plouin pour la mise à disposition des demiproduits de Durrenentzen. Sans son accord pour la réalisation d'analyses archéométriques passant nécessairement par un tronçonnage d'objets archéologiques, cette étude interdisciplinaire novatrice n'aurait pu être réalisée. Nos remerciements vont à la société Crelier SA qui a procédé gracieusement au tronçonnage des objets au jet d'eau. Nous remercions également l'équipe du LMC14 (LSCE) pour la réalisation des mesures radiocarbones sur ARTEMIS et nos collègues du CEB-IRAMAT pour leur accueil et leur aide à l'ICP-MS à ablation laser. 


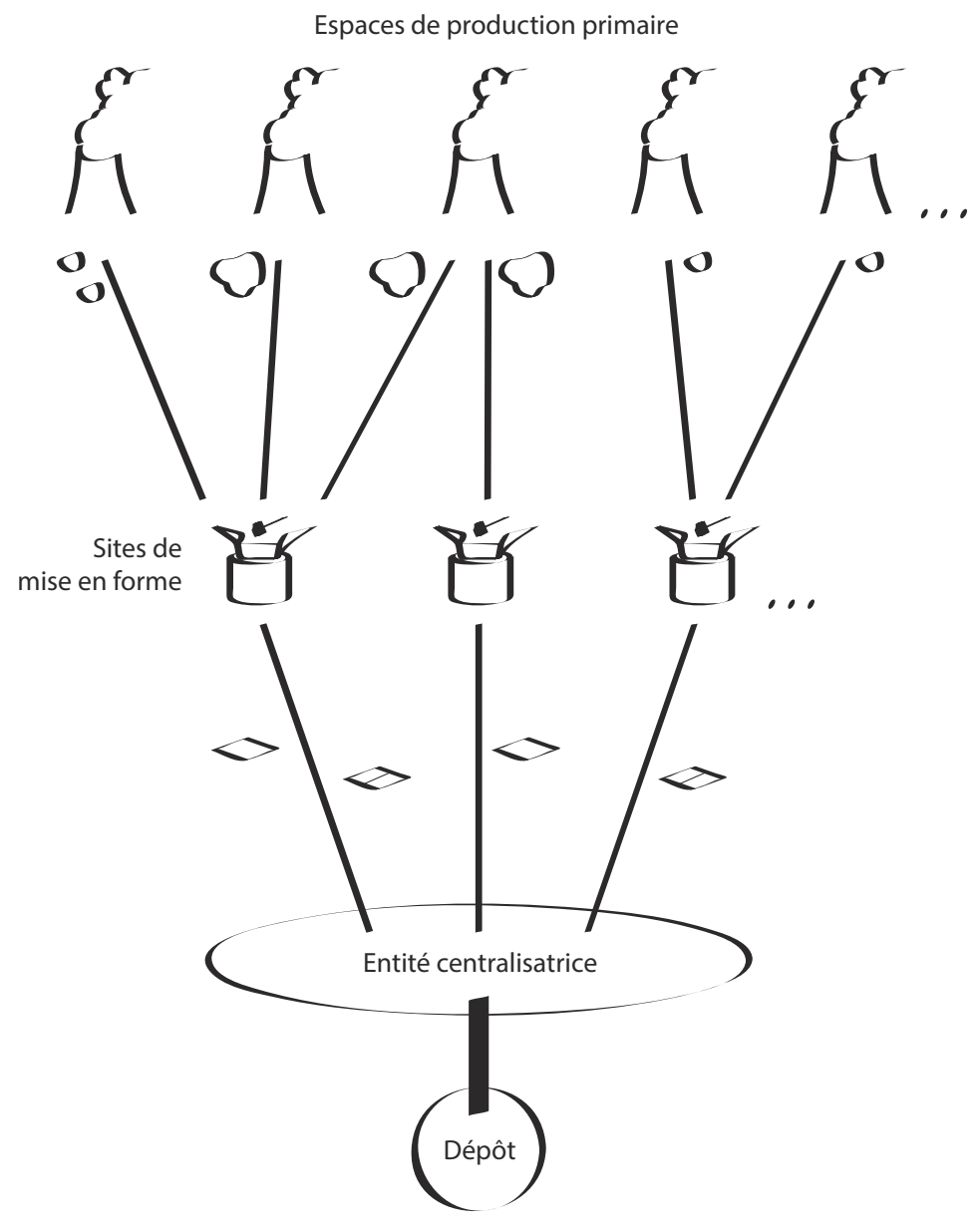
Plusieurs espaces de production caractérisés par : - plusieurs traditions techniques
* masses de tailles variées
* alliage fer/carbone hétérogène
* présence variable de phosphore
* compaction initiale possiblement différente
- des signatures géochimiques différentes
* ressources géologiques différentes

Possibles intermédiaires commerciaux :

Un site peut acquérir des masses provenant de plusieurs espaces de production primaire
Plusieurs sites de mise en forme caractérisés par : - des standards morphologiques partagés
* forme bipyramidée des demi-produits malgré une mise en forme par soudure de deux masses - des standards métrologiques communs
* poids et tailles quasi identiques
* Cassures des pointes pour se conformer au standard
- de légères variations de mise en forme
* arrêtes centrales plus ou moins marquées
* arrêtes latérales plus ou moins rectilignes

Une entité centralisatrice caractérisée par un pouvoir suffisamment fort pour :

- centraliser plus de $300 \mathrm{~kg}$ de fer

* faible quantité de fer retrouvée sur les sites

d'habitat contemporains

* le fer est presque exclusivement destiné aux élites sociales (parure et armement)

- unir plusieurs sites de mise en forme autour d'un standard métrologique

* possible mise en place d'étalon

- sortir une telle masse de métal du circuit économique

* démonstration de pouvoir, pratique rituelle,...

Figure 12 : Modèle théorique des relations entre les différents acteurs impliqués dans la mise en place du dépôt de Durrenentzen. Figure 12: Theoretical model showing relations between the various actors involved in the implementation of the Durrenentzen deposit.

\section{Bibliographie}

Augier L., Balzer I., Bardel D., Deffressigne S., Bertrand E., Fleischer F., Hopert-Hagmann S., Lndolt M., Mennessier-Jouannet C., Mège C., Roth-Zehner M., Saurel M., Tappert C., Thierrin-Michael G., Tikonoff N., 2013. La céramique faconnée au tour : témoin privilégié de la diffusion des techniques au Hallstatt D2-D3 et à La Tène A-B1. In A. Colin, F. Verdin (ed.), L'âge du Fer en Aquitaine et sur ses marges. Mobilité des hommes, diffusion des idées, circulation des biens dans l'espace européen de l'âge du Fer. Actes du XXXV colloque de l'Association Française pour l'Étude de l'Âge du Fer, Bordeaux 2-5 juin 2011. Fédération Aquitania, Bordeaux, coll «Aquitania; 30 », p. 563-594.

Balzer I., 2009. Chronologisch-chorologische Untersuchung des späthallstatt- und frühlatènezeitlichen „Fürstensitzes“ auf dem
Münsterberg von Breisach (Grabungen 1980-1986). KonradTheiss Verlag, Stuttgart, 316 p. (Materialhefte zur Archäologie in Baden-Württemberg 84).

Bauvais S., Fluzin P., 2006. Réflexion sur l'organisation technicosociale des activités de forge à La Tène finale dans l'Aisne (02). Revue d'Archéométrie, 30, p. 25-43.

Bauvais S., 2008. Du prestige à la proto-industrie : évolution des pratiques sidérurgiques au second âge du fer dans le Nord du Bassin parisien. The Arkeotek Journal, 2, (4) : [http://www. thearkeotekjournal.org/tdm/Arkeotek/fr/archives/2008/4Bauvais. $x m l$.

Bauvais S., Fluzin P., 2009. Archaeological and Archeometrical Approaches of the Chaîne Opératoire in Iron and Steelmaking: Methodology for a Regional Evolution Study. In V. Roux, S. Rosen (ed.), Techniques and People: anthropological perspectives on technology in the archaeology of the proto-historic and 
early historic periods in the Southern Levant. Paris, Éditions De Bocard, p. 157-178.

Bauvais S., Schwab R., Brauns M., Dillmann P., 2011. Circulation of Iron Products in the Iron Age of Eastern France and Southern Germany: multidisciplinary and methodological approaches towards the provenance of ancient iron, a DFGANR project. In A. Hauptmann, D. Modarressi-Tehrani, M. Prange (ed.), Archaeometallurgy in Europe III. Bochum, Deutsches Bergbau-Museum Bochum, coll. " Metalla, Sonderhelf; 4 ", p. 95-96.

Bauvais S., Dillmann P., Disser A., Leroy S., Berranger M., Pagès G., Vega E., 2015. Circulation of Iron Products in the Iron-Age of Eastern France and Southern Germany: Multidisciplinary and Methodological Approaches Towards the Provenance of Ancient Iron (CIPIA). Rapport final de programme ANR-DFG. Agence Nationale de la Recherche, LAPA-IRAMAT, Paris, Saclay, 161 p.

Berranger M., Bauvais S., Fluzin P., 2007. "Socket bars": multidisciplinary results (archaeology and archaeometry) on a specific iron semi-product in the northern Parisian basin (France). In Archaeometallurgy in Europe 2007. International conference in Grado-Aquilea (Italy). Publication sur CD.

Berranger M., Fluzin P., 2012. From iron to semi-product: quality and circulation of materials during the Iron Age in France. Archaeometry, 54, (4), p. 664-684.

Berranger M., 2014. Le fer, entre matière première et moyen d'échange, en France du VII au Ir s. av. J.-C. Approches interdisciplinaires. Dijon, Éditions Universitaires de Dijon, coll. «Art, archéologie, histoire ", $382 \mathrm{p}$.

Berranger M. (dir.), 2015. La sidérurgie en Bourgogne et en Franche-Comté avant le haut-fourneau. Organisation et circulation des productions. Projet collectif de recherche, rapport d'activité 2015. LMC-IRAMAT, Service Régional de l'Archéologie Bourgogne-Franche-Comté, Belfort, Besançon, 234 p.

Biellmann P., 1986. Les lingots de fer de Durrenentzen. Annuaire de la société d'histoire de la Hardt et du Ried, 1, p. 11-18.

Carrara S., Dubreucq E., Pescher B., 2013. La fabrication des fibules à timbale comme marqueur des contacts et des transferts technologiques au cours du Ha D-LT A1. Nouvelles données d'après les sites de Bourges, Lyon et Plombières-les-Dijon. In A. Colin, F. Verdin (ed.), L'âge du Fer en Aquitaine et sur ses marges. Mobilité des hommes, diffusion des idées, circulation des biens dans l'espace européen de l'âge du Fer. Actes du XXXV colloque de l'Association Française pour l'Étude de l'Âge du Fer, Bordeaux 2-5 juin 2011. Bordeaux, Fédération Aquitania, coll. «Aquitania, supplément; 30 », p. 595-608.

Charlton M.F., 2015. The last frontier in "sourcing": the hopes, constraints and future for iron provenance research. Journal of Archaeological Science, 56, p. 210-220.
Coustures M.-P., Béziat D., Tollon F., Domergue C., Long L., Rebiscoul A., 2003. The use of trace element analysis of entrapped slag inclusions to establish ore - Bar iron links: examples from two Gallo-Roman iron-making sites in France (Les Martys, Montagne Noire, and Les Ferrys, Loiret). Archaeometry, 45, (4), p. 599-613.

Coustures M.-P., Dieudonné-Glad N., Dillmann P., Béziat D., 2014. Tentative chemical characterization of a Roman smelting workshop (Oulches, France): from the ore to the finished product. In B. Cech, Th. Rehren (ed.), Early Iron in Europe, Montagnac, Éditions Monique-Mergoil, coll. "Monographies Instrumentum; 50 », p. 93-115.

Desaulty A.-M., Dillmann P., L'Héritier M., Mariet C., Gratuze B., Joron J.-L., Fluzin P., 2009. Does it come from the Pays de Bray? Examination of an origin hypothesis for the ferrous reinforcements used in French medieval churches using major and trace element analyses. Journal of Archaeological Science, 36, (10), p. 2445-2462.

Dillmann P., Leroy S., Disser A., Bauvais S., Vega E., Fluzin P., 2015. Dernières avancées autour des études sur la production, la circulation et la datation des métaux ferreux archéologiques. Les nouvelles de l'archéologie, 138, p. 28-34.

Dillmann P., L'Héritier M., 2007. Slag inclusion analyses for studying ferrous alloys employed in French medieval buildings: supply of materials and diffusion of smelting processes. Journal of Archaeological Science, 34, (11), p. 1810-1823.

Disser A., 2014. Production et circulation du fer en Lorraine ( $\mathrm{VI}^{\mathrm{e}}$ s. av. J.-C.-Xve s. apr. J.-C.). Thèse de doctorat, Université de Technologie de Belfort-Montbéliard, France, 1038 p.

Disser A., Dillmann P., Bourgain C., L'Héritier M., Vega E., Bauvais S., Leroy M., 2014. Iron reinforcements in Beauvais and Metz Cathedrals: from bloomery or finery? The use of logistic regression for differentiating smelting processes. Journal of Archaeological Science, 42, (1), p. 315-333.

Disser A., Dillmann P., Leroy M., L'Héritier M., Bauvais S., FuuzIN P., 2016. Iron supply for the building of Metz cathedral: new methodological development for provenance studies and historical considerations. Archaeometry, 59, (3), p. 493-510.

Dubreucq E., 2013. Métal des premiers celtes, productions métalliques sur les habitats des provinces du Hallstatt centre-occidental. Dijon, Éditions Universitaires de Dijon, coll. «Art, archéologie, histoire ", $450 \mathrm{p}$.

Fluzin P., 2002. La chaîne opératoire en sidérurgie : matériaux archéologiques et procédés. Apports des études métallographiques. In H. Bocoum (ed.), Aux origines de la métallurgie du fer en Afrique. Une ancienneté méconnue. Afrique de l'Ouest et Afrique centrale. Paris, Éditions UNESCO, p. 59-91.

Fluzin P., Bauvais S., Berranger M., Pagès G., Dillmann P., 2011. The multidisciplinary approach (archaeology and archaeometry) to bloomsmithing activities in France: 
examples of results from the last twenty years. In J. Hosek, H. Cleere, L. Mihok (dir.), The archaeometallurgy of Iron - Recent Developments in Archaeological and Scientific Research. Institute of Archarology of the ASCR, Prague, p. 223-236.

Fluzin P., Berranger M., Bauvais S., Pagès G., Dillmann P., 2012. An archaeological and archaeometrical approach of ferrous semi-product: typology, quality and circulation. In C. Cuccini (dir.), Acta Mineraria et Metallurgica - Studi in onore di Marco Tizzoni. Bergame, Notizie Archeologiche Bergomensi, p. 195-204.

Fournier L., Milcent P.-Y., 2007. Actualité des recherches sur l'économie du fer protohistorique dans la Région Centre. In P.-Y. Milcent (ed.), L'économie du fer protohistorique : de la production à la consommation du métal. XXVIII colloque AFAEF, Toulouse 20-23 mai 2004. Bordeaux, Fédération Aquitania, coll. «Aquitania; 14/2 », p. 85-105.

Galili E., Bauvais S., Rosen B., Dillmann P., 2015. Cargoes of iron semi-products recovered from shipwrecks off the carmel coast, israel. Archaeometry, 57, (3), p. 505-535.

Kleeman O., 1981. Les lingots de fer bipyramidaux courts et épais : les lingots du type Colmar. Études offertes à J.-J. Hatt. Revue archéologique de l'Est, 32, p. 109-119.

Kunnl G., 2015. Alsace, Haut Rhin (68), Durrenentzen, Rue des Commandos - Lotissement "Oben an den Gaerten". Un deuxième dépôt de demi-produits de fer de l'âge du Fer à Durrenentzen. Rapport de Diagnostic. Strasbourg/Dijon, SRA Alsace/INRAP Grand-Est-Sud, 47 p.

Leroy S., Cohen S.X., Verna C., Gratuze B., Téreygeol F., Fluzin P., Bertrand L., Dillmann P., 2012a. The medieval iron market in Ariège (France). Multidisciplinary analytical approach and multivariate analyses. Journal of Archaeological Science, 39, (4), p. 1080-1093.

Leroy S., Delque-Kolic E., Dumoulin J.-P., Moreau C., Dillmann P., 2012b. Datation radiocarbone des alliages ferreux anciens. Actes du colloque Sciences des matériaux du patrimoine culturel - 2, Paris, 20-21 novembre 2012, p. 57-63 [http://www. culturecommunication.gouv.fr/Politiques-ministerielles/RechercheEnseignement-superieur-Technologies/Actualites/Actes-du-colloqueSciences-des-materiaux-du-patrimoine-culturel].

Leroy S., Dillmann P., Disser A., L'Heritier M., Bauvais S., FLUZIN P., 2014. Provenance et circulation des alliages ferreux. In P. Dillmann, L. Bellot-Gurlet (dir.), Circulation et provenance des matériaux dans les sociétés anciennes. Paris, Éditions des Archives contemporaines, p. 73-101.

Leroy S., Delque-Kolic E., Dumoulin J.-P., Moreau C., Dillmann P., 2015a. Consolidation or initial design?
Radiocarbon dating of ancient iron alloys sheds light on the reinforcements of French Gothic Cathedrals. Journal of Archaeological Science, 53, (0), p. 190-201.

Leroy S., Hendrickson M., Delque-Kolic E., Vega E., Dillmann P., 2015b. First Direct Dating for the Construction and Modification of the Baphuon Temple Mountain in Angkor, Cambodia. PLoS ONE, 10, (11) : e0141052. doi:10.1371/journal.pone.0141052.

PAGÈs G., 2010. Artisanat et économie du fer en France méditerranéenne de l'Antiquité au début du Moyen Âge : une approche interdisciplinaire. Montagnac, Éditions Monique-Mergoil, coll. "Monographies Instrumentum; 37 », 315 p.

Pagès G., Dillmann P., Fluzin P., Long L., 2011. A study of the roman iron bars of Saintes-Maries-de-la-Mer (Bouchesdu-Rhône, France). A proposal for a comprehensive metallographic approach. Journal of Archaeological Science 38, (6), p. 1234-1252.

Pleiner R., Bjorkman J., 1974. The Assyrian Iron Age: The History of Iron in the Assyrian Civilization. Proceedings of the American Philosophical Society, 118, (3), p. 283-313.

Pleiner R., 2006. Iron in archaeology. The European blacksmiths. Archeologicky ustav, Prague, $384 \mathrm{p}$.

Ramsey C.B., LeE S., 2013. Recent and planned developments of the program OxCal. Radiocarbon, 55 (2-3), p. 3-4.

Reimer P.J., Bard E., Bayliss A., Beck J.W., Blackwell P.G., Bronk Ramsey C., Buck C.E., Cheng H., Edwards R.L., Friedrich M., Grootes P.M., Guilderson T.P., Haflidason H., Hajdas I., Hatté C., Heaton T.J., Hoffmann D.L., Hogg A.G., Hughen K.A., Kaiser K.F., Kromer B., Manning S.W., Niu M., Reimer R.W., Richards D.A., Scott E.M., Southon J.R., Staff R.A., Turney C.S.M., van Der Plicht J., 2013. IntCal13 and Marine13 radiocarbon age calibration curves 0-50,000 years cal BP. Radiocarbon 55, (4), p. $1869-1887$.

Senn M., Kraack M., Flisch A., Wichser A., Obrist M., 2014. An aspect of the celtic iron trade: the spitzbarren. The deposit from Bellmund (canton Bern, Switzerland). In B. Cech, T. Rehren (ed.), Early iron in Europe. Éditions Monique Mergoil, Montagnac, « Monographie Instrumentum; 50 », p. 147-160.

Thouvenin A., 1984. Lingots de fer gaulois et techniques de forge. Revue archéologique de l'est et du centre est, 35, (3-4), p. 368-372.

Vega E., Dillmann P., Fluzin P., 2002. Contribution à l'étude de fers phosphoreux en sidérurgie ancienne. Revue d'Archéométrie, 26, p. 197-208. 